\title{
Elucidating Reactivity Differences in Palladium-Catalyzed Coupling Processes: The Chemistry of Palladium Hydrides
}

\author{
Ivory D. Hills and Gregory C. Fu* \\ Department of Chemistry, Massachusetts Institute of Technology, \\ Cambridge, Massachusetts 02139
}

\section{Supporting Information}

\section{General}

All reactions were carried out under an atmosphere of nitrogen or argon in oven-dried glassware with magnetic stirring, unless otherwise indicated.

$\mathrm{Pd}\left(\mathrm{P}(t-\mathrm{Bu})_{3}\right)_{2}$ (Strem and Johnson Matthey), $\mathrm{Pd}_{2}(\mathrm{dba})_{3}$ (Aldrich), $\mathrm{PCy}_{3}$ (Strem), and $\mathrm{P}(t-\mathrm{Bu})_{3}(\mathrm{FMC})$ were used as received. $\mathrm{Pd}\left(\mathrm{PCy}_{3}\right)_{2}$ (Strem) was purified by recrystallization from toluene/methanol.

THF was purified under argon by passage through a neutral alumina column. Dioxane (Aldrich; Sure Seal), $\mathrm{Cs}_{2} \mathrm{CO}_{3}$ (Strem), phenyl boronic acid (Frontier Scientific), and $1.0 \mathrm{M} \mathrm{HCl}$ in $\mathrm{Et}_{2} \mathrm{O}$ (Aldrich) were used as received. $\mathrm{Cy}_{2} \mathrm{NMe}$ (Aldrich), $n$-butyl vinyl ether (Aldrich), and 4'-chloroacetophenone (Aldrich) were de-gassed by bubbling dry argon through the liquids.

${ }^{1} \mathrm{H}$ and ${ }^{13} \mathrm{C}$ NMR resonances are referenced to the solvent. ${ }^{31} \mathrm{P}$ NMR resonances are referenced to external $85 \% \mathrm{H}_{3} \mathrm{PO}_{4}$. 


\section{Preparation of $\mathrm{L}_{2} \mathrm{PdHCl}$ Complexes}

General procedure. In a glovebox, $\mathrm{HCl}\left(1.0 \mathrm{M}\right.$ in $\mathrm{Et}_{2} \mathrm{O} ; 1.1$ equiv) was added dropwise over 2 minutes to a stirring $0.1 \mathrm{M}$ solution of the $\mathrm{PdL}_{2}$ complex (1.0 equiv) in THF. Upon the initial addition of $\mathrm{HCl}$, the reaction mixture darkened; however, after approximately 15 minutes of stirring at room temperature, the solution turned lightyellow. The reaction mixture was stirred for a total of $1 \mathrm{~h}$, and then it was filtered. The solvent and the excess $\mathrm{HCl}$ were removed under vacuum, and the resulting solid was washed with pentanes and then dried under vacuum.

$\left(\mathbf{P}(t-B u)_{3}\right)_{2} \mathbf{P d H C l}$ [63166-71-2]. The general procedure was followed, using 250 $\mathrm{mg}(0.49 \mathrm{mmol})$ of $\mathrm{Pd}\left(\mathrm{P}(t-\mathrm{Bu})_{3}\right)_{2}$ and $0.54 \mathrm{~mL}$ of $1.0 \mathrm{M} \mathrm{HCl}$ in $\mathrm{Et}_{2} \mathrm{O}$. The product (260 $\mathrm{mg}, 97 \%$ yield) was isolated as an off-white powder.

$1 \mathrm{H} \mathrm{NMR}\left(300 \mathrm{MHz}, \mathrm{THF}-\mathrm{d}_{8}\right) \delta-16.41\left(\mathrm{t}, 1 \mathrm{H},{ }^{2} \mathrm{~J}_{\mathrm{H}-\mathrm{P}}=6.0 \mathrm{~Hz}\right), 1.57(\mathrm{~m}, 54 \mathrm{H}) .{ }^{13 \mathrm{C}}$ NMR (300 MHz, THF) $\delta 32.7\left(t,{ }^{2} \mathrm{~J}_{\mathrm{C}-\mathrm{P}}=11.7 \mathrm{~Hz}\right), 39.6\left(\mathrm{t},{ }^{1} \mathrm{~J}_{\mathrm{C}-\mathrm{P}}=14.4 \mathrm{~Hz}\right) .31 \mathrm{P}$ NMR (300 MHz, THF) $\delta$ 83.1. FTIR (thin film) 2901, 1477, 1393, 1361, 1170, $808 \mathrm{~cm}^{-1}$.

$\left.(\mathbf{P C})_{3}\right)_{2} \mathbf{P d H C l}$ [28016-71-9]. The general procedure was followed, using $300 \mathrm{mg}$ $(0.45 \mathrm{mmol})$ of $\mathrm{Pd}\left(\mathrm{PC}_{3}\right)_{2}$ and $0.50 \mathrm{~mL}$ of $1.0 \mathrm{M} \mathrm{HCl}$ in $\mathrm{Et}_{2} \mathrm{O}$. The product $(310 \mathrm{mg}, 98 \%$ yield) was isolated as a gray powder.

1H NMR (300 MHz, THF-d 8$) \delta-14.61\left(\mathrm{t}, 1 \mathrm{H},{ }^{2} \mathrm{~J}_{\mathrm{H}-\mathrm{P}}=4.2 \mathrm{~Hz}\right), 1.29(\mathrm{~m}, 18 \mathrm{H}), 1.55(\mathrm{~m}$, 12H), $1.73(\mathrm{~m}, 18 \mathrm{H}), 2.15(\mathrm{~m}, 12 \mathrm{H}), 2.14(\mathrm{~m}, 6 \mathrm{H}) .13 \mathrm{C} \mathrm{NMR} \mathrm{(300} \mathrm{MHz,} \mathrm{THF)} \delta 26.8,27.7$ $\left(\mathrm{t},{ }^{2} \mathrm{~J}_{\mathrm{C}-\mathrm{P}}=23.4 \mathrm{~Hz}\right), 30.7,34.4\left(\mathrm{t},{ }^{1} \mathrm{~J}_{\mathrm{C}-\mathrm{P}}=40.2 \mathrm{~Hz}\right) .{ }^{31 P} \mathrm{NMR}(300 \mathrm{MHz}, \mathrm{THF}) \delta 42.6$. FTIR (thin film) 2926, 2850, 1446, 1266, 1174, 1070, 1004, 888, $849 \mathrm{~cm}^{-1}$. 


\section{Reactivity Studies}

Procedure for eq 3 and eq 4 . In a nitrogen-filled glovebox, $\mathrm{Pd}_{2}(\mathrm{dba})_{3}(3.6 \mathrm{mg}$, $0.018 \mathrm{mmol}$ ) and 4 '-chloroacetophenone $(66 \mathrm{mg}, 0.66 \mathrm{mmol})$ were added to a Tefloncapped vial equipped with a stir bar. The Brønsted base $\left(0.66 \mathrm{mmol}\right.$ of either $\mathrm{Cs}_{2} \mathrm{CO}_{3}$ (220 mg) or $\left.\mathrm{Cy}_{2} \mathrm{NMe}(130 \mathrm{mg})\right), \mathrm{P}(t-\mathrm{Bu})_{3}$ (59 mg, $\left.0.30 \mathrm{mmol}\right)$, $n$-butyl vinyl ether (8.2 $\mathrm{mg}, 0.0090 \mathrm{mmol}$ ), tetradecane (59 mg, $0.30 \mathrm{mmol}$; internal standard for GC analysis), and dioxane $(0.60 \mathrm{~mL})$ were added via syringe. The reaction mixture was stirred at room temperature. To measure the yields of the Heck arylation product, aliquots were removed after 4 hours and after 32 hours of reaction, filtered through a plug of silica with copious washings with $\mathrm{Et}_{2} \mathrm{O}$, and analyzed by GC.

31P NMR analysis (eq 4). Aliquots were removed after 4 hours and transferred directly to an NMR tube for 31P NMR analysis. For the reaction in the presence of $\mathrm{Cy}_{2} \mathrm{NMe}$, a resonance (>98\%) at $86 \mathrm{ppm}$ was observed; this coincides with the resonance for $\operatorname{Pd}\left(\mathrm{P}(t-\mathrm{Bu})_{3}\right)_{2}$. For the reaction in the presence of $\mathrm{Cs}_{2} \mathrm{CO}_{3}$, a resonance $(>98 \%)$ at $83 \mathrm{ppm}$ was observed; this coincides with the resonance for $\left(\mathrm{P}(t-\mathrm{Bu})_{3}\right)_{2} \mathrm{PdHCl}$ (see spectra below).

\section{Reference 31P NMR shifts ( $)$.}

\begin{tabular}{lccc}
\cline { 2 - 4 } & $\mathrm{L}_{2} \mathrm{PdHCl}$ & $\mathrm{PdL}_{2}$ & $\mathrm{~L}$ \\
\cline { 2 - 4 } $\mathrm{P}(t-\mathrm{Bu})_{3}$ & 83.1 & 85.9 & 63.4 \\
$\mathrm{PCy}_{3}$ & 42.6 & 39.7 & 10.9 \\
\hline
\end{tabular}


31P NMR spectra for Heck reactions (eq 4).

Reaction with $\mathrm{Cs}_{2} \mathrm{CO}_{3}$ as the Bronsted base

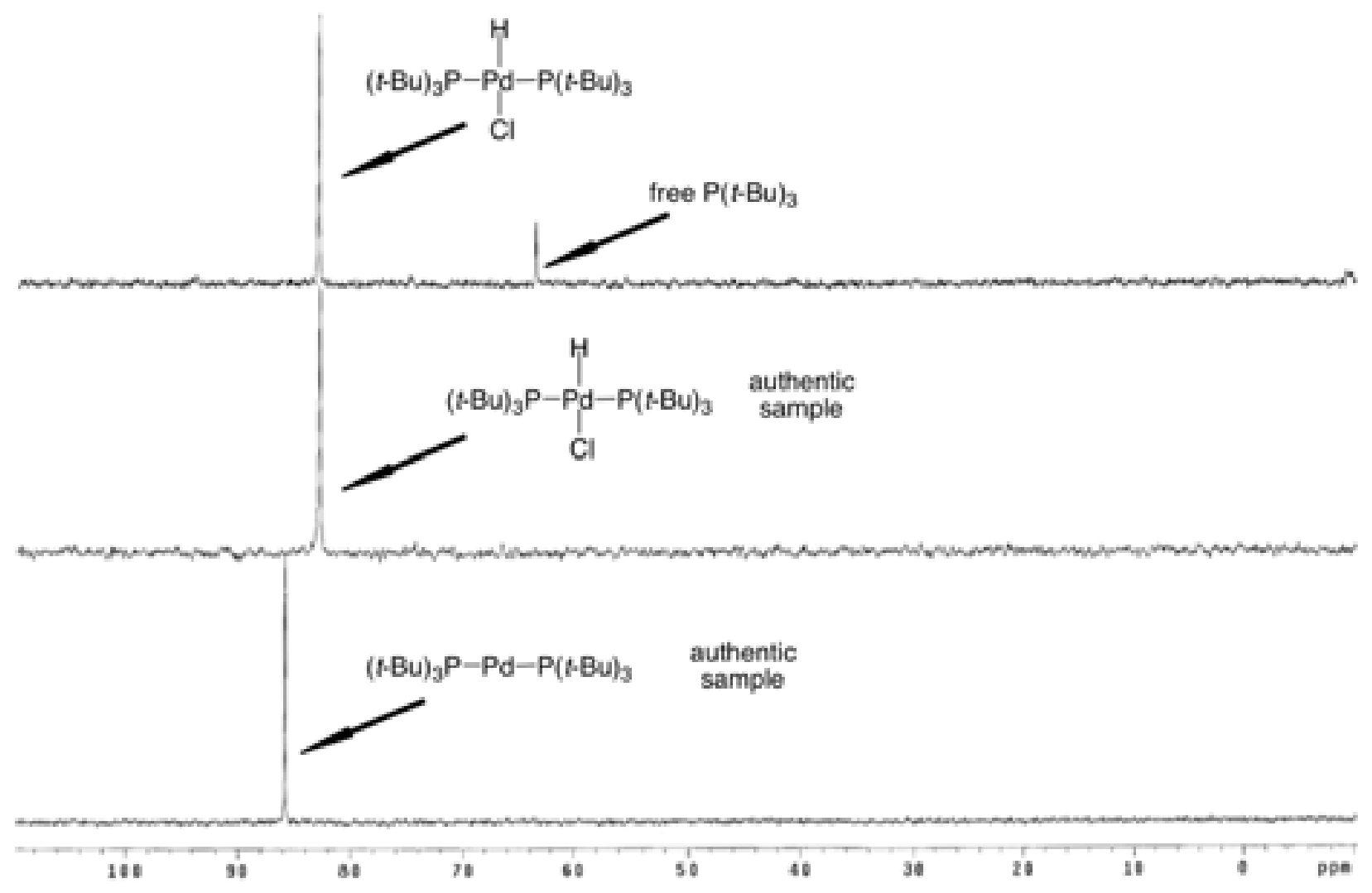




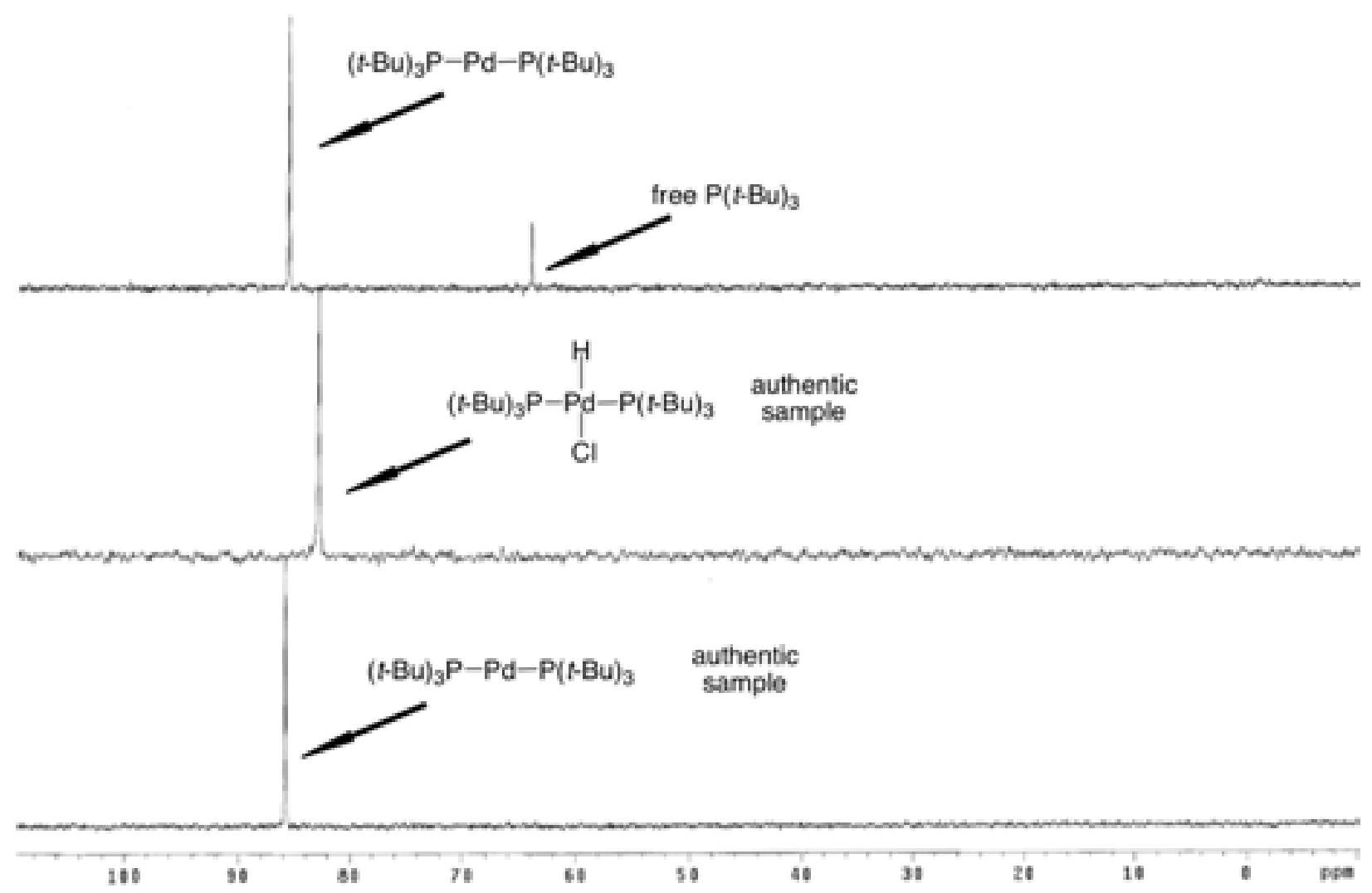

Procedure for eq 5, starting with $\mathrm{L}_{2} \mathrm{PdHCl}$. In a glovebox, $\mathrm{L}_{2} \mathrm{PdHCl}$ (1.0 equiv) was added to a vial and dissolved in dioxane. Then, $\mathrm{Cy}_{2} \mathrm{NMe}$ (35 equiv) was added to the reaction mixture, and the entire contents of the vial were transferred to an NMR tube for 31P NMR analysis.

Eq 5, $\mathbf{L}=\mathbf{P}(t-B u)_{3}$. The general procedure was followed, using $\left(\mathrm{P}(t-\mathrm{Bu})_{3}\right)_{2} \mathrm{PdHCl}$ (9.9 mg, $0.018 \mathrm{mmol}), \mathrm{Cy}_{2} \mathrm{NMe}(120 \mathrm{mg}, 0.63 \mathrm{mmol})$, and dioxane $(0.60 \mathrm{~mL}) .31 \mathrm{P}$ NMR analysis was performed 30 minutes and 5.5 hours after the start of the reaction. After 30 minutes, the ratio of $\left(\mathrm{P}(t-\mathrm{Bu})_{3}\right)_{2} \mathrm{PdHCl}$ and $\mathrm{Pd}\left(\mathrm{P}(t-\mathrm{Bu})_{3}\right)_{2}$ was $36: 64$, with a trace $(<5 \%)$ of free $\mathrm{P}(t-\mathrm{Bu})_{3}$. After 5.5 hours, the ratio of $\left(\mathrm{P}(t-\mathrm{Bu})_{3}\right)_{2} \mathrm{PdHCl}$ and $\mathrm{Pd}\left(\mathrm{P}(t-\mathrm{Bu})_{3}\right)_{2}$ was $<2:>98$, with a trace $(<5 \%)$ of free $\mathrm{P}(t-\mathrm{Bu})_{3}$ (see spectra below). 
Eq 5, $\mathrm{L}=\mathbf{P C} y_{3}$. The general procedure was followed, using $\left(\mathrm{PCy}_{3}\right)_{2} \mathrm{PdHCl}(13.0$ $\mathrm{mg}, 0.018 \mathrm{mmol}), \mathrm{Cy}_{2} \mathrm{NMe}(120 \mathrm{mg}, 0.63 \mathrm{mmol})$, and dioxane $(0.60 \mathrm{~mL}) .31 \mathrm{P} \mathrm{NMR}$ analysis was performed 30 minutes and 5.5 hours after the start of the reaction. After 30 minutes, the ratio of $\left(\mathrm{PCy}_{3}\right)_{2} \mathrm{PdHCl}$ and $\mathrm{Pd}\left(\mathrm{PCy}_{3}\right)_{2}$ was $>98:<2$, and no free $\mathrm{PCy}_{3}$ was observed. After 5.5 hours, the ratio of $\left(\mathrm{P}(t-\mathrm{Bu})_{3}\right)_{2} \mathrm{PdHCl}$ and $\mathrm{Pd}\left(\mathrm{P}(t-\mathrm{Bu})_{3}\right)_{2}$ remained at $>98:<2$, and no free РСуз was observed (see spectra below).

31P NMR spectra for reactions of $\mathrm{L}_{2} \mathrm{PdHCl}$ with $\mathrm{Cy}_{2} \mathrm{NMe}$ (eq 5).

Reaction with $P(t-B u)_{3}$ as the ligand

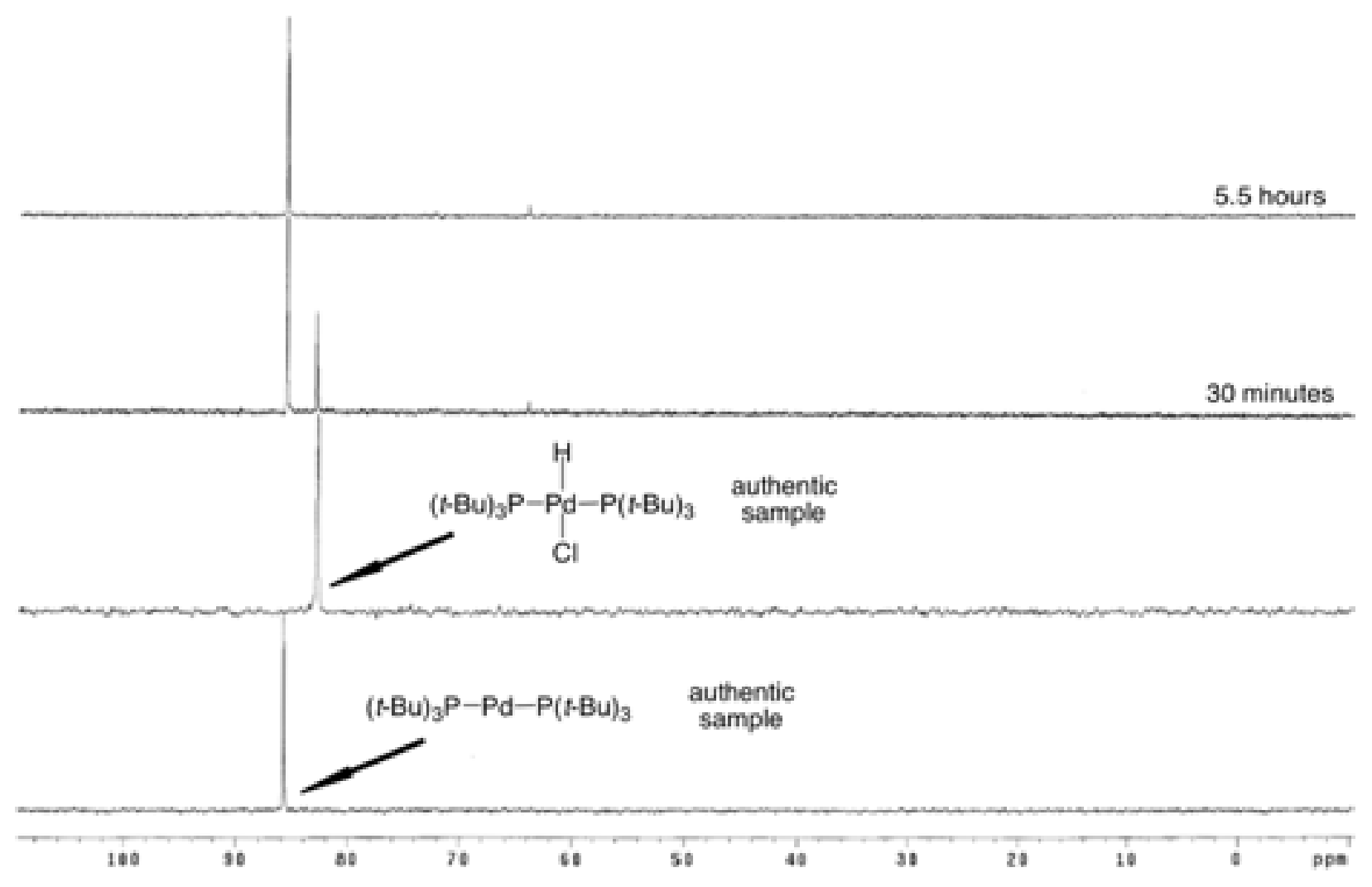


Reaction with $\mathrm{PC}_{3}$ as the ligand

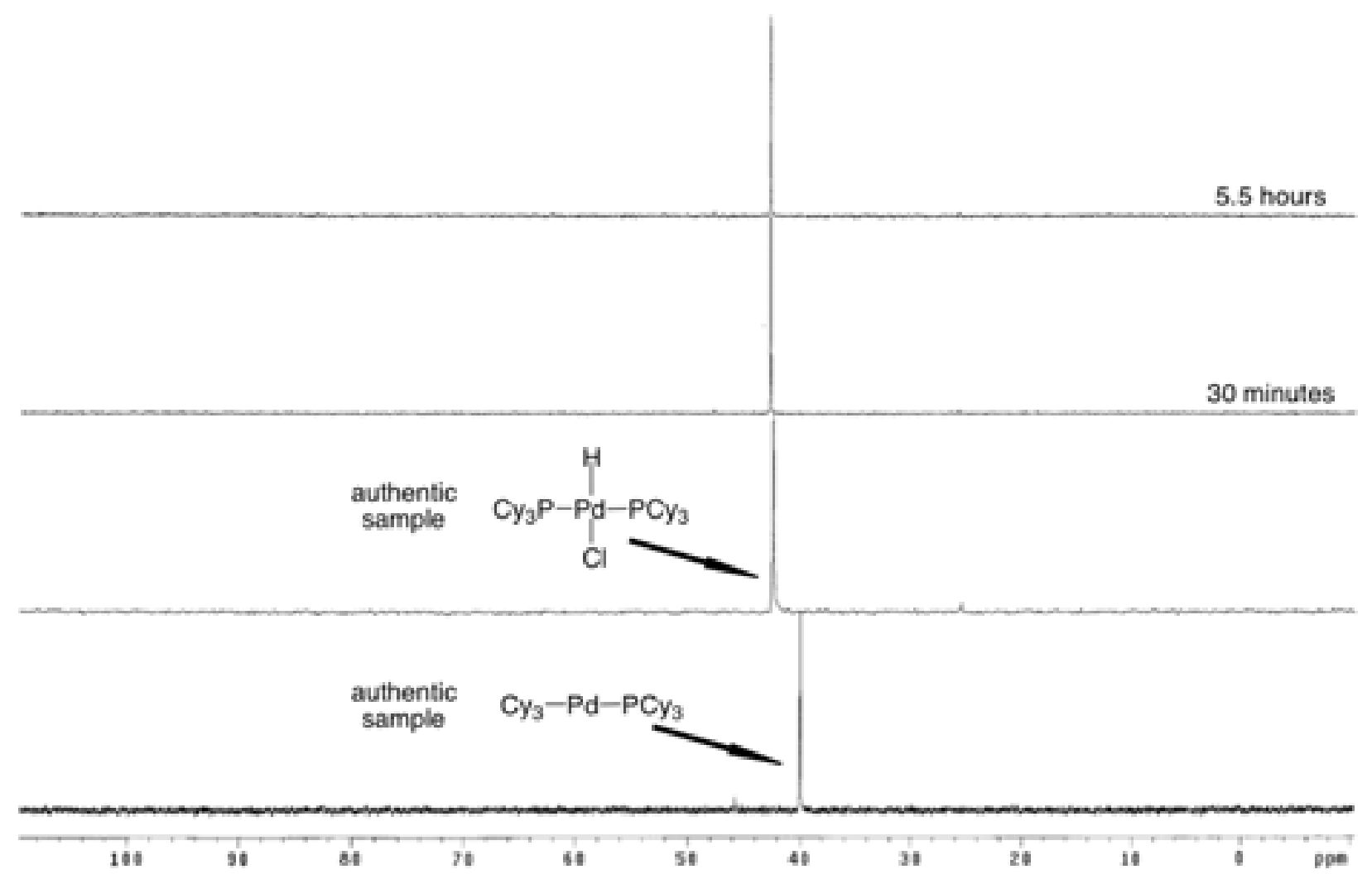

Procedure for eq 5, starting with $\mathrm{PdL}_{2}$. In order to verify that the ratios of $\mathrm{L}_{2} \mathrm{PdHCl}: \mathrm{PdL}_{2}$ in eq 5 are equilibrium ratios, the equilibrium was approached from the opposite direction (starting from the right-hand side of eq 5).

In a glovebox, $\mathrm{PdL}_{2}$ (1.0 equiv) was added to a vial and dissolved in dioxane. Then, $\left[\mathrm{Cy}_{2} \mathrm{NHMe}\right] \mathrm{Cl}$ (1.0 equiv) was added, followed by $\mathrm{Cy}_{2} \mathrm{NMe}$ (34 equiv). The entire contents of the vial were transferred to an NMR tube for 31P NMR analysis.

Eq $5, \mathbf{L}=\mathbf{P}(t-\mathbf{B u})_{3}$. The general procedure was followed, using $\operatorname{Pd}\left(\mathrm{P}(t-\mathrm{Bu})_{3}\right)_{2}(9.2$ $\mathrm{mg}, 0.018 \mathrm{mmol}),\left[\mathrm{Cy}_{2} \mathrm{NHMe}\right] \mathrm{Cl}(4.2 \mathrm{mg}, 0.018 \mathrm{mmol}), \mathrm{Cy}_{2} \mathrm{NMe}(120 \mathrm{mg}, 0.61 \mathrm{mmol})$, and dioxane $(0.60 \mathrm{~mL})$. The entire contents of the vial were transferred after 30 minutes to an NMR tube and analyzed by $31 \mathrm{P}$ NMR. The ratio of $\left(\mathrm{P}(t-\mathrm{Bu})_{3}\right)_{2} \mathrm{PdHCl}: \mathrm{Pd}(\mathrm{P}(t-$ $\left.\mathrm{Bu})_{3}\right)_{2}$ was $<2:>98$. 
Eq 5, L = PCу $\mathbf{y}_{3}$. The general procedure was followed, using $\mathrm{Pd}\left(\mathrm{PC}_{3}\right)_{2}(12 \mathrm{mg}$, $0.018 \mathrm{mmol}),\left[\mathrm{Cy}_{2} \mathrm{HNMe}\right] \mathrm{Cl}(4.2 \mathrm{mg}, 0.018 \mathrm{mmol}), \mathrm{Cy}_{2} \mathrm{NMe}(120 \mathrm{mg}, 0.61 \mathrm{mmol})$, and dioxane $(0.60 \mathrm{~mL})$. The entire contents of the vial were transferred after 30 minutes to an NMR tube and analyzed by $31 \mathrm{P} \mathrm{NMR}$. The ratio of $(\mathrm{PCy})_{2}{ }_{2} \mathrm{PdHCl}: \mathrm{Pd}(\mathrm{PCy} 3)_{2}$ was $>98:<2$. 


\section{Equation 6.}

$$
\begin{gathered}
\text { Rate }=\mathrm{k}\left[\mathrm{HClPdL}_{2}\right] \\
\frac{d\left[\mathrm{HClPdL}_{2}\right]}{d t}=-k\left[\mathrm{HClPdL}_{2}\right] \stackrel{\text { integration }}{\longrightarrow} \int_{t_{0}}^{t} \frac{1}{\left[\mathrm{HClPdL}_{2}\right]} d\left[\mathrm{HClPdL}_{2}\right]=-\int_{t_{0}}^{t} k d t \\
\ln \left(\frac{\left[\mathrm{HClPdL}_{2}\right]_{t}}{\left[\mathrm{HClPdL}_{2}\right]_{t_{0}}}\right)=-k\left(t-t_{0}\right) \stackrel{\text { simplification }}{\longrightarrow} \ln \left(\frac{\left[\mathrm{HClPdL}_{2}\right]}{\left[\mathrm{HClPdL}_{2}\right]_{0}}\right)=-k t
\end{gathered}
$$

Thus, if the reductive elimination obeys the above rate law, a plot of the conversion as a function of time (see above) will furnish a line, the slope of which is the first-order rate constant, $\mathrm{k}$.

Using the Eyring equation:

$$
k=\frac{\kappa T}{h} e^{\frac{-\Delta G^{\sharp}}{R T}}
$$

The free energy of activation $\left(\Delta \mathrm{G}^{\ddagger}\right)$ can be solved for by rearrangement:

$$
\begin{gathered}
\Delta G^{\ddagger}=-R T \ln \left(\frac{\mathrm{kh}}{\kappa T}\right) \\
R=1.9872 \mathrm{cal} \bullet \mathrm{mol}^{-1} \bullet \mathrm{K}^{-1} \\
T=\text { temperature in Kelvin } \\
k=\text { rate constant } \\
h=6.62608 \times 10^{-34} \mathrm{~J} \bullet \mathrm{s} \\
\kappa=1.3806 \times 10^{-23} \mathrm{~J} \bullet \mathrm{K}^{-1}
\end{gathered}
$$


General procedure for kinetics studies. In a glovebox, $\left(\mathrm{P}(t-\mathrm{Bu})_{3}\right)_{2} \mathrm{PdHCl}(9.9$ $\mathrm{mg}, 0.018 \mathrm{mmol}, 1.0$ equiv) was added to a vial, dissolved in dioxane $(0.20 \mathrm{~mL})$, and transferred to an NMR tube. The vial was washed with dioxane $(2 \times 0.20 \mathrm{~mL})$, and the washings were transferred to the NMR tube, giving an overall concentration of $0.03 \mathrm{M}$ in the palladium complex. The tube was sealed with a Teflon screw-cap and cooled to $15{ }^{\circ} \mathrm{C}$ in the spectrometer. After the temperature had reached equilibrium, $\mathrm{Cy}_{2} \mathrm{NMe}$ (3.4 $\mu \mathrm{L}, 3.5 \mathrm{mg}, 0.018 \mathrm{mmol}, 1.0$ equiv) was added to the NMR tube through the Teflon septum.

The reaction was monitored by $31 \mathrm{P}$ NMR at $15^{\circ} \mathrm{C}$, and the percent conversion of $\left(\mathrm{P}(t-\mathrm{Bu})_{3}\right)_{2} \mathrm{PdHCl}$ to $\mathrm{Pd}\left(\mathrm{P}(t-\mathrm{Bu})_{3}\right)_{2}$ was determined by integrating the two resonances (attempts to use an internal standard led to anomalous results). 
Support for a first-order reaction (eq 6). A reaction was set up and monitored according to the general procedure. The data were plotted using the kinetic analysis for a first-order reaction outlined above, which furnished a linear plot (see graph below). These data indicate that $\mathrm{k}=0.000155 \mathrm{~s}^{-1}$.

$y=-0.081909-0.0001548 x R=0.99805$

\section{XII-79A}

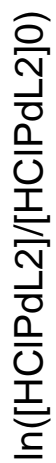

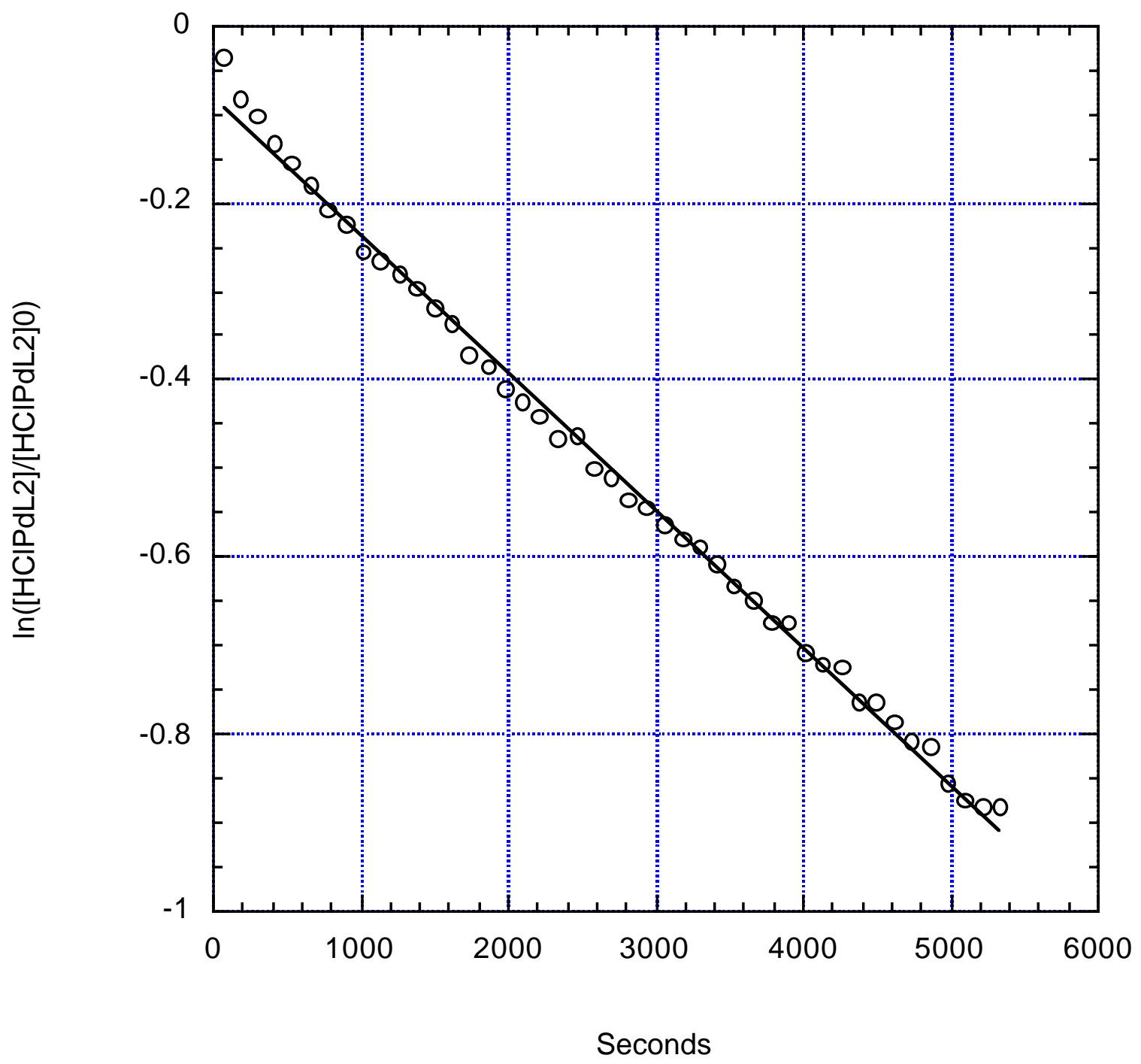


Order of the reaction in $\mathrm{Cy}_{2} \mathrm{NMe}$ (eq 6). Three reactions were set up and monitored according to the general procedure. The three reactions had 1.0, 2.0, or 4.0 equiv of $\mathrm{Cy}_{2} \mathrm{NMe}$. The data (see graph below) indicate that $\mathrm{k}=0.000155 \mathrm{~s}^{-1}, 0.000162 \mathrm{~s}^{-1}$, and $0.000167 \mathrm{~s}^{-1}$ for $1.0,2.0$, and 4.0 equiv of $\mathrm{Cy}_{2} \mathrm{NMe}$, respectively. These values are consistent with a zero-order dependence on $\mathrm{Cy}_{2} \mathrm{NMe}$.

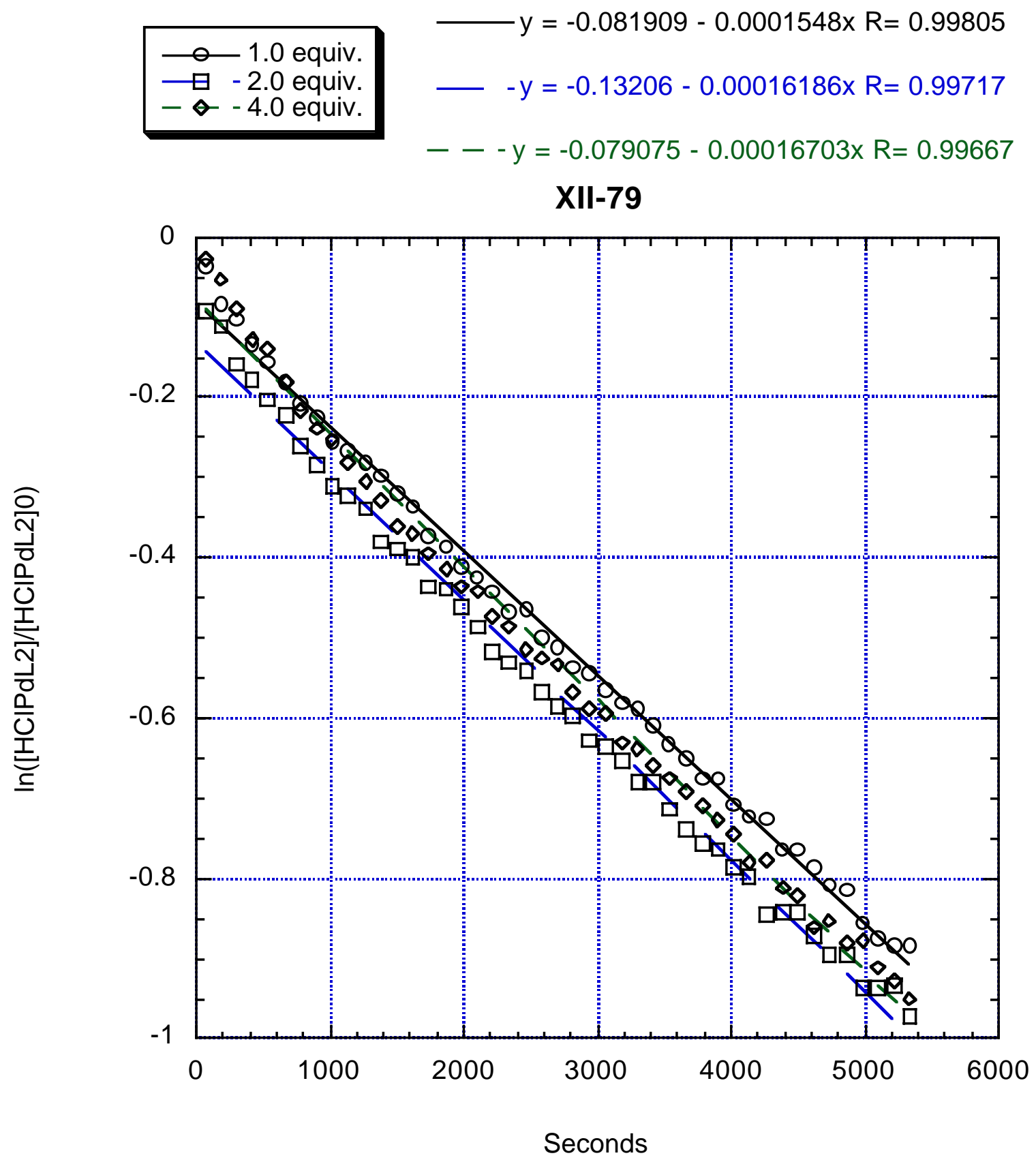


Effect of added $\mathrm{P}(t-\mathrm{Bu})_{3}$ (eq 6). Three reactions were set up and monitored according to the general procedure. The three reactions had $0.0,2.0$, or 4.0 equiv of added $\mathrm{P}(t-\mathrm{Bu})_{3}$. These data indicate that $\mathrm{k}=0.000155 \mathrm{~s}^{-1}, 0.000111 \mathrm{~s}^{-1}$, and $0.000107 \mathrm{~s}^{-1}$ for $0.0,2.0$, and 4.0 equiv of added $\mathrm{P}(t-\mathrm{Bu})_{3}$, respectively. These values are consistent with an inhibitory effect of added $\mathrm{P}(t-\mathrm{Bu})_{3}$ (see graph below).

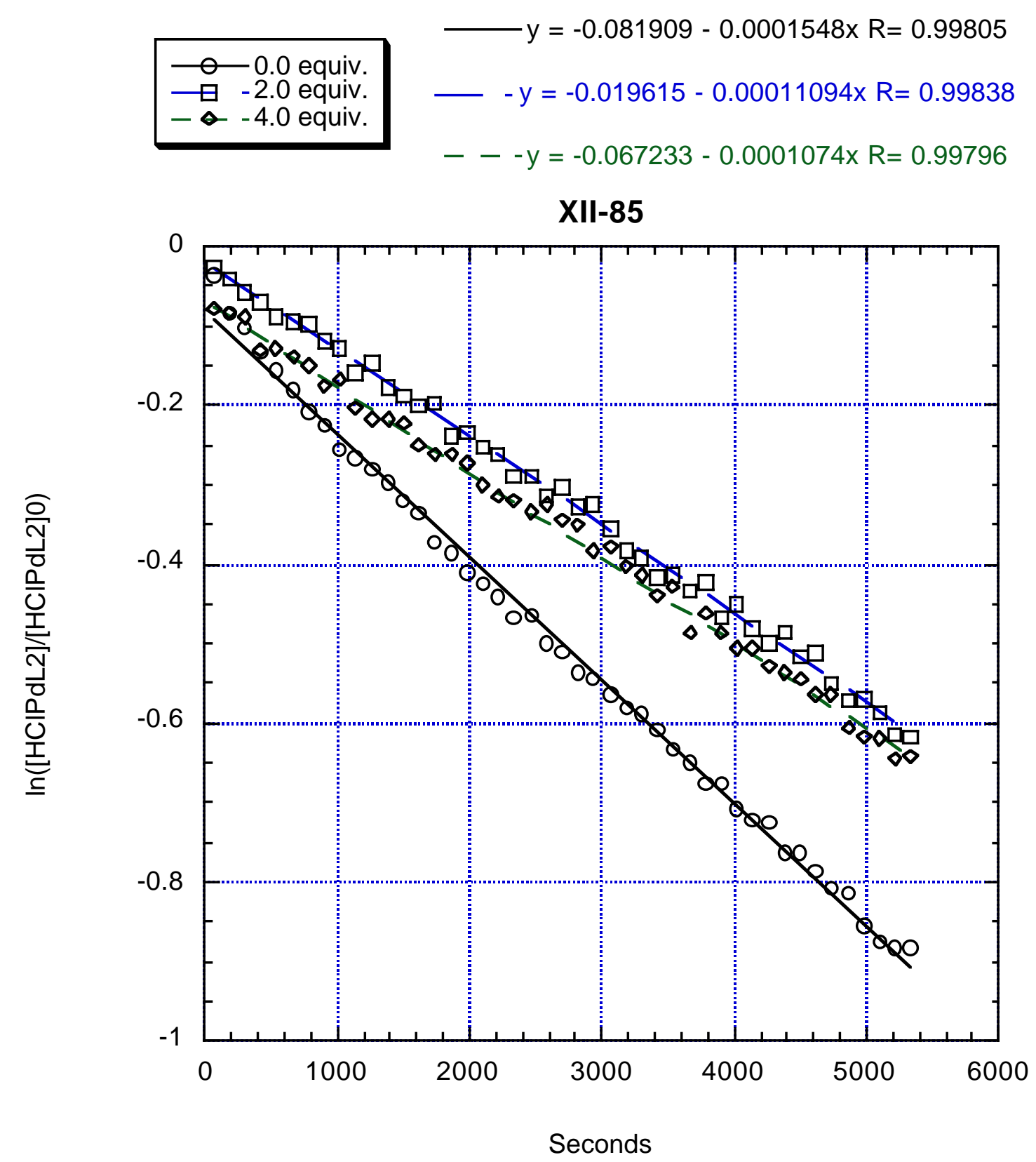


Activation energy for reductive elimination (eq 5). Two reactions were set up at $20{ }^{\circ} \mathrm{C}$ using 35 equiv of $\mathrm{Cy}_{2} \mathrm{NMe}$ (mimicking the conditions found in a $\mathrm{Pd} / \mathrm{P}(t-\mathrm{Bu})_{3^{-}}$ catalyzed Heck reaction) and monitored according to the general procedure. These data indicate that $\mathrm{k}=0.000143 \mathrm{~s}^{-1}$ and $0.000129 \mathrm{~s}^{-1}$ for run 1 and run 2 , respectively. These rate constants correspond to $\Delta \mathrm{G}^{\ddagger}=22.3 \mathrm{kcal} / \mathrm{mol}$ and $22.4 \mathrm{kcal} / \mathrm{mol}$, respectively.
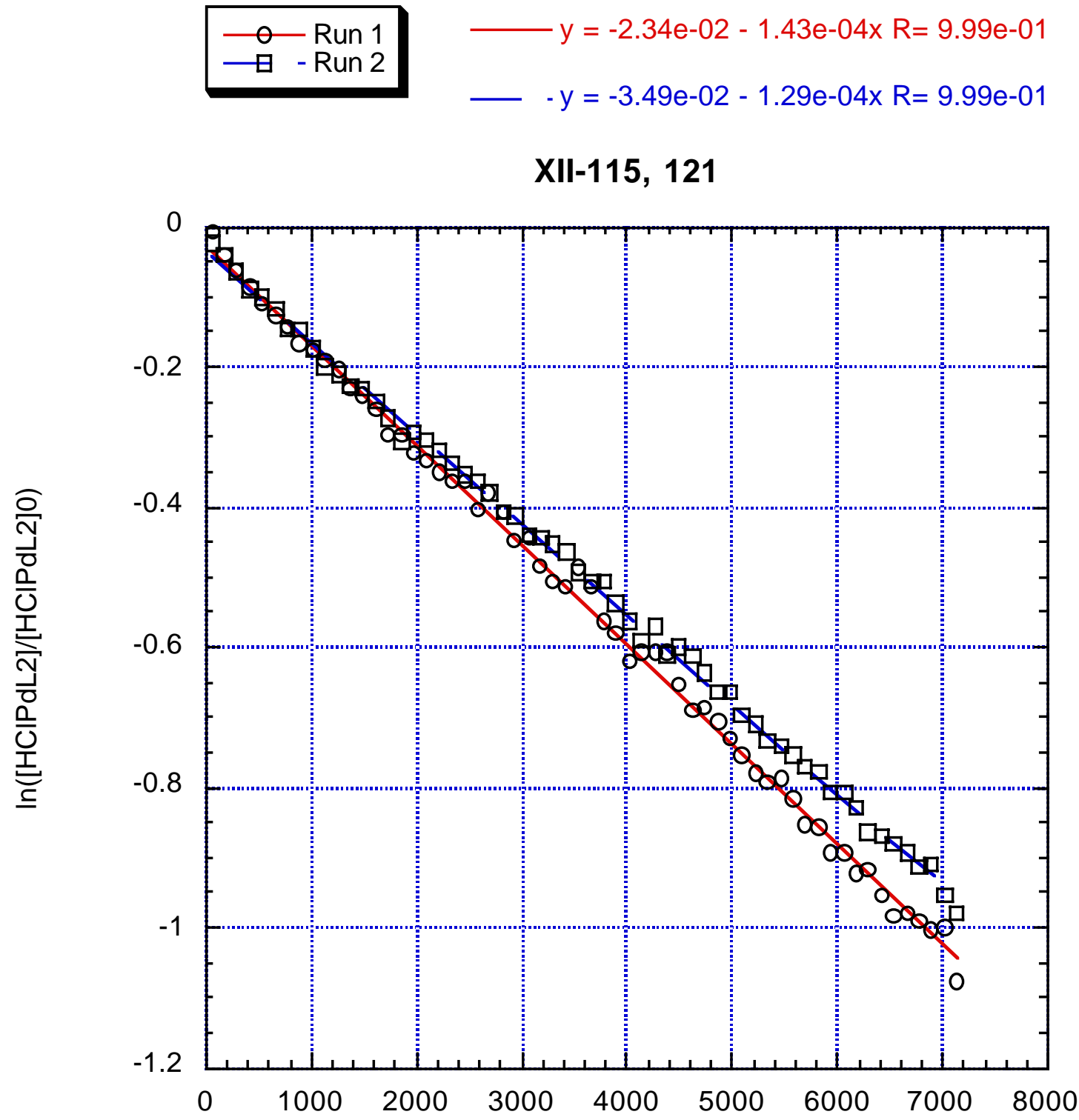

Seconds 


\section{X-ray Crystallographic Data}<smiles>[13CH3][Pb]([13CH3])(Cl)Cl</smiles>

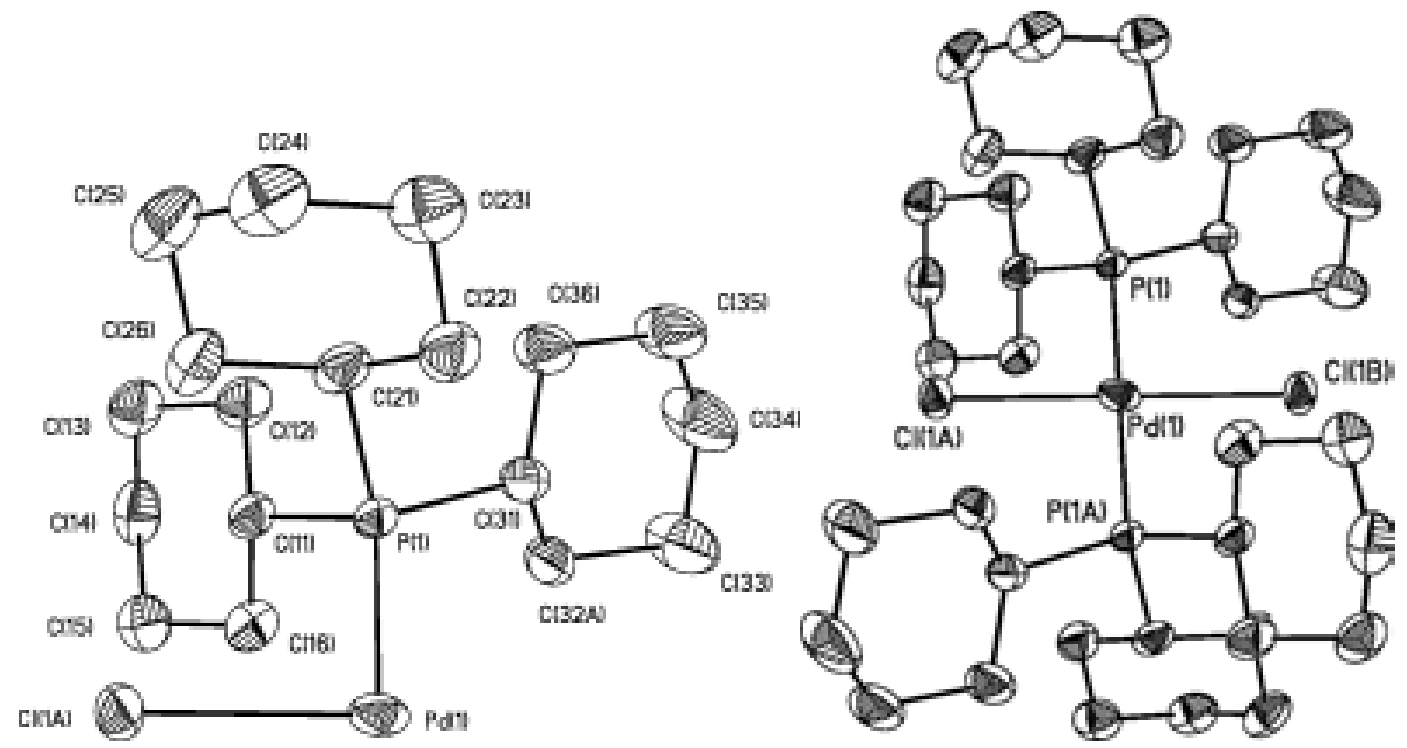

A light-gray solution of $\mathbf{1}$ in $\mathrm{Et}_{2} \mathrm{O}$ was prepared. Crystals suitable for X-ray structural analysis were obtained by solvent evaporation.

A colorless block of dimensions $0.28 \times 0.19 \times 0.16 \mathrm{~mm}^{3}$ was mounted under STP and transferred to a Bruker AXS/CCD three-circle diffractometer ( $\chi$ fixed at $54.78^{\circ}$ ) equipped with a cold stream of $\mathrm{N}_{2}$ gas. An initial unit cell was determined by harvesting reflections I > $20 \sigma(\mathrm{I})$ from $45 \times 10$-s frames of $0.30^{\circ} \omega$ scan data with monochromated Mo $\mathrm{K}_{\alpha}$ radiation $(\lambda=0.71073 \AA)$. The cell thus determined was triclinic.

A hemisphere of data was then collected using $\omega$ scans of $0.30^{\circ}$ and 30 -s frames. The raw data frames were integrated using the Bruker program SAINT+ for NT version 
6.01. The data that were collected (4013 total reflections, 3242 unique, $\left.R_{\text {int }}=0.0229\right)$ had the following Miller index ranges: -10 to 10 in $h,-10$ to 11 in $k$, and -11 to 11 in 1 . No absorption correction was performed.

All aspects of the solution and refinement were handled by SHELXTL NT version 5.10. The structure was solved by direct methods in the triclinic space group P-1, a = 9.8843(5) $\AA ; b=10.2473(5) \AA ; c=10.6255(6) \AA ; \alpha=66.6700(10)^{\circ} ; \beta=70.1810(1)^{\circ} ; \gamma=$ $89.9180(10)^{\circ}$, and refined using standard difference Fourier techniques.

For the P-1 solution, full-matrix least-squares refinement (2596 data for 197 parameters) on $\mathrm{F}^{2}$ yielded residuals of $\mathrm{R}_{1}$ and $\mathrm{wR}_{2}$ of 0.0371 and 0.0884 for data $\mathrm{I}>2 \sigma$ (I), and 0.0397 and 0.0905, respectively, for all data. During the final refinement, all nonhydrogen atoms were treated anisotropically. Hydrogen atoms were included in calculated positions and refined isotropically on a riding model. Residual electron density amounted to a maximum of $0.557 \mathrm{e} / \AA^{3}$ and a minimum of $-1.033 \mathrm{e} / \AA^{3}$.

The chlorine, $\mathrm{Cl}(\mathbf{1 A})$, has been refined with a partial occupancy and through symmetry generates $\mathbf{C l}(\mathbf{1 A}) \#$ ( $~ 50 \%$ in each position). There is disorder in $\mathbf{C}(\mathbf{3 2})$, represented by $\mathbf{C}(32 \mathrm{~A})$ and $\mathbf{C}(32 \mathrm{~B})$. The cyclohexyl ring incorporating $\mathrm{C}(32)$ is partially in a chair $(\sim 80 \%)$ and a boat $(\sim 20 \%)$ conformation. The metal-hydride has not been located and is represented graphically in Figure 2 merely for illustrative purposes. 
Tables 7-12 provide the full crystallographic data for the X-ray structure solved in P-1.

Table 7. Crystal data and structure refinement for 04014IH p-1.

Identification code

Empirical formula

Formula weight

Temperature

Wavelength

Crystal system

Space group

Unit cell dimensions

Volume

Z

Density (calculated)

Absorption coefficient

$\mathrm{F}(000)$

Crystal size

Theta range for data collection

Index ranges

Reflections collected

Independent reflections

Completeness to theta $=23.24^{\circ}$

Refinement method

Data / restraints / parameters

Goodness-of-fit on $\mathrm{F}^{2}$

Final $\mathrm{R}$ indices [I $>2$ sigma(I)]

$\mathrm{R}$ indices (all data)

Largest diff. peak and hole
04014IH p-1

C36 H66 Cl P2 Pd

702.68

193(2) K

$0.71073 \AA$

Triclinic

P-1

$$
\begin{array}{ll}
\mathrm{a}=9.8843(5) \AA & \alpha=66.6700(10)^{\circ} . \\
\mathrm{b}=10.2473(5) \AA & \beta=70.1810(10)^{\circ} . \\
\mathrm{c}=10.6255(6) \AA & \gamma=89.9180(10)^{\circ} .
\end{array}
$$

918.64(8) $\AA^{3}$

1

$1.270 \mathrm{Mg} / \mathrm{m}^{3}$

$0.687 \mathrm{~mm}^{-1}$

375

$0.28 \times 0.19 \times 0.16 \mathrm{~mm}^{3}$

2.19 to $23.24^{\circ}$.

$-10<=\mathrm{h}<=10,-10<=\mathrm{k}<=11,-11<=\mathrm{l}<=11$

2596

$2596[\mathrm{R}(\mathrm{int})=0.0000]$

$98.7 \%$

Full-matrix least-squares on $\mathrm{F}^{2}$

2596 / 242 / 197

1.042

$\mathrm{R} 1=0.0371, \mathrm{wR} 2=0.0884$

$\mathrm{R} 1=0.0397, \mathrm{wR} 2=0.0905$

0.557 and -1.033 e. $\AA^{-3}$ 
Table 8. Atomic coordinates $\left(x 10^{4}\right)$ and equivalent isotropic displacement parameters $\left(\AA^{2} \times 10^{3}\right)$ for $\mathrm{p}-1$. U(eq) is defined as one third of the trace of the orthogonalized Uij tensor.

\begin{tabular}{lcccc}
\hline & $\mathrm{x}$ & $\mathrm{y}$ & $\mathrm{z}$ & $\mathrm{U}(\mathrm{eq})$ \\
\hline $\mathrm{Pd}(1)$ & 0 & 0 & 0 & $54(1)$ \\
$\mathrm{P}(1)$ & $-2005(1)$ & $-330(1)$ & $2063(1)$ & $41(1)$ \\
$\mathrm{C}(11)$ & $-1649(4)$ & $-1164(4)$ & $3785(3)$ & $43(1)$ \\
$\mathrm{C}(12)$ & $-2903(4)$ & $-1352(5)$ & $5202(4)$ & $56(1)$ \\
$\mathrm{C}(13)$ & $-2371(5)$ & $-1793(5)$ & $6481(4)$ & $63(1)$ \\
$\mathrm{C}(14)$ & $-1717(5)$ & $-3144(5)$ & $6685(4)$ & $65(1)$ \\
$\mathrm{C}(15)$ & $-496(5)$ & $-3010(5)$ & $5285(5)$ & $66(1)$ \\
$\mathrm{C}(16)$ & $-994(4)$ & $-2544(4)$ & $3982(4)$ & $58(1)$ \\
$\mathrm{C}(21)$ & $-2546(4)$ & $1418(4)$ & $2014(4)$ & $45(1)$ \\
$\mathrm{C}(22)$ & $-2830(4)$ & $2270(4)$ & $602(4)$ & $54(1)$ \\
$\mathrm{C}(23)$ & $-3301(4)$ & $3710(4)$ & $535(5)$ & $60(1)$ \\
$\mathrm{C}(24)$ & $-2184(4)$ & $4593(4)$ & $691(4)$ & $62(1)$ \\
$\mathrm{C}(25)$ & $-1929(5)$ & $3759(4)$ & $2100(4)$ & $65(1)$ \\
$\mathrm{C}(26)$ & $-1442(4)$ & $2325(4)$ & $2174(4)$ & $56(1)$ \\
$\mathrm{C}(31)$ & $-3680(4)$ & $-1331(4)$ & $2260(5)$ & $55(1)$ \\
$\mathrm{C}(32 \mathrm{~A})$ & $-3672(5)$ & $-2938(6)$ & $2692(8)$ & $66(2)$ \\
$\mathrm{C}(32 \mathrm{~B})$ & $-3640(20)$ & $-2170(30)$ & $1380(30)$ & $104(8)$ \\
$\mathrm{C}(33)$ & $-4908(5)$ & $-3500(7)$ & $2327(9)$ & $127(3)$ \\
$\mathrm{C}(34)$ & $-6355(5)$ & $-3283(6)$ & $3245(9)$ & $116(2)$ \\
$\mathrm{C}(35)$ & $-6390(4)$ & $-1799(6)$ & $3028(7)$ & $91(2)$ \\
$\mathrm{C}(36)$ & $-5131(4)$ & $-1129(5)$ & $3198(5)$ & $64(1)$ \\
$\mathrm{Cl}(1 \mathrm{~A})$ & $1638(2)$ & $797(2)$ & $1035(2)$ & $49(1)$ \\
& & & & \\
\hline
\end{tabular}


Table 9. Bond lengths $[\AA]$ and angles $\left[^{\circ}\right]$ for $04014 \mathrm{IH}$ p-1.

\begin{tabular}{lc}
\hline $\mathrm{Pd}(1)-\mathrm{P}(1) \# 1$ & $2.3058(8)$ \\
$\mathrm{Pd}(1)-\mathrm{P}(1)$ & $2.3058(8)$ \\
$\mathrm{Pd}(1)-\mathrm{Cl}(1 \mathrm{~A}) \# 1$ & $2.5337(16)$ \\
$\mathrm{Pd}(1)-\mathrm{Cl}(1 \mathrm{~A})$ & $2.5337(16)$ \\
$\mathrm{P}(1)-\mathrm{C}(11)$ & $1.844(3)$ \\
$\mathrm{P}(1)-\mathrm{C}(31)$ & $1.853(3)$ \\
$\mathrm{P}(1)-\mathrm{C}(21)$ & $1.854(3)$ \\
$\mathrm{C}(11)-\mathrm{C}(16)$ & $1.522(5)$ \\
$\mathrm{C}(11)-\mathrm{C}(12)$ & $1.532(4)$ \\
$\mathrm{C}(12)-\mathrm{C}(13)$ & $1.525(5)$ \\
$\mathrm{C}(13)-\mathrm{C}(14)$ & $1.496(6)$ \\
$\mathrm{C}(14)-\mathrm{C}(15)$ & $1.520(6)$ \\
$\mathrm{C}(15)-\mathrm{C}(16)$ & $1.525(5)$ \\
$\mathrm{C}(21)-\mathrm{C}(26)$ & $1.529(5)$ \\
$\mathrm{C}(21)-\mathrm{C}(22)$ & $1.529(5)$ \\
$\mathrm{C}(22)-\mathrm{C}(23)$ & $1.528(5)$ \\
$\mathrm{C}(23)-\mathrm{C}(24)$ & $1.520(5)$ \\
$\mathrm{C}(24)-\mathrm{C}(25)$ & $1.510(6)$ \\
$\mathrm{C}(25)-\mathrm{C}(26)$ & $1.526(5)$ \\
$\mathrm{C}(31)-\mathrm{C}(32 \mathrm{~B})$ & $1.492(13)$ \\
$\mathrm{C}(31)-\mathrm{C}(36)$ & $1.512(5)$ \\
$\mathrm{C}(31)-\mathrm{C}(32 \mathrm{~A})$ & $1.526(6)$ \\
$\mathrm{C}(32 \mathrm{~A})-\mathrm{C}(33)$ & $1.573(6)$ \\
$\mathrm{C}(32 \mathrm{~B})-\mathrm{C}(33)$ & $1.586(15)$ \\
$\mathrm{C}(33)-\mathrm{C}(34)$ & $1.507(7)$ \\
$\mathrm{C}(34)-\mathrm{C}(35)$ & $1.447(6)$ \\
$\mathrm{C}(35)-\mathrm{C}(36)$ & $1.519(6)$ \\
$\mathrm{P}(1) \# 1-\mathrm{Pd}(1)-\mathrm{P}(1)$ & $180.00(6)$ \\
$\mathrm{P}(1) \# 1-\mathrm{Pd}(1)-\mathrm{Cl}(1 \mathrm{~A}) \# 190.50(4)$ \\
$\mathrm{P}(1)-\mathrm{Pd}(1)-\mathrm{Cl}(1 \mathrm{~A}) \# 1$ & $89.50(4)$ \\
$\mathrm{P}(1) \# 1-\mathrm{Pd}(1)-\mathrm{Cl}(1 \mathrm{~A})$ & $89.50(4)$ \\
$\mathrm{P}(1)-\mathrm{Pd}(1)-\mathrm{Cl}(1 \mathrm{~A})$ & $90.50(4)$ \\
$\mathrm{Cl}(1 \mathrm{~A}) \# 1-\mathrm{Pd}(1)-\mathrm{Cl}(1 \mathrm{~A}) 180.00(8)$ \\
$\mathrm{C}(11)-\mathrm{P}(1)-\mathrm{C}(31)$ & $109.42(18)$ \\
&
\end{tabular}




$\begin{array}{ll}\mathrm{C}(11)-\mathrm{P}(1)-\mathrm{C}(21) & 104.15(15) \\ \mathrm{C}(31)-\mathrm{P}(1)-\mathrm{C}(21) & 103.56(15) \\ \mathrm{C}(11)-\mathrm{P}(1)-\mathrm{Pd}(1) & 112.80(11) \\ \mathrm{C}(31)-\mathrm{P}(1)-\mathrm{Pd}(1) & 114.93(12) \\ \mathrm{C}(21)-\mathrm{P}(1)-\mathrm{Pd}(1) & 111.06(11) \\ \mathrm{C}(16)-\mathrm{C}(11)-\mathrm{C}(12) & 110.8(3) \\ \mathrm{C}(16)-\mathrm{C}(11)-\mathrm{P}(1) & 114.2(2) \\ \mathrm{C}(12)-\mathrm{C}(11)-\mathrm{P}(1) & 116.2(2) \\ \mathrm{C}(13)-\mathrm{C}(12)-\mathrm{C}(11) & 110.2(3) \\ \mathrm{C}(14)-\mathrm{C}(13)-\mathrm{C}(12) & 110.8(3) \\ \mathrm{C}(13)-\mathrm{C}(14)-\mathrm{C}(15) & 112.1(3) \\ \mathrm{C}(14)-\mathrm{C}(15)-\mathrm{C}(16) & 111.7(3) \\ \mathrm{C}(11)-\mathrm{C}(16)-\mathrm{C}(15) & 110.3(3) \\ \mathrm{C}(26)-\mathrm{C}(21)-\mathrm{C}(22) & 110.3(3) \\ \mathrm{C}(26)-\mathrm{C}(21)-\mathrm{P}(1) & 113.4(3) \\ \mathrm{C}(22)-\mathrm{C}(21)-\mathrm{P}(1) & 110.3(2) \\ \mathrm{C}(23)-\mathrm{C}(22)-\mathrm{C}(21) & 111.8(3) \\ \mathrm{C}(24)-\mathrm{C}(23)-\mathrm{C}(22) & 110.9(3) \\ \mathrm{C}(25)-\mathrm{C}(24)-\mathrm{C}(23) & 110.6(3) \\ \mathrm{C}(24)-\mathrm{C}(25)-\mathrm{C}(26) & 111.6(3) \\ \mathrm{C}(25)-\mathrm{C}(26)-\mathrm{C}(21) & 111.2(3) \\ \mathrm{C}(32 \mathrm{~B})-\mathrm{C}(31)-\mathrm{C}(36) & 119.6(9) \\ \mathrm{C}(32 \mathrm{~B})-\mathrm{C}(31)-\mathrm{C}(32 \mathrm{~A}) & 50.7(13) \\ \mathrm{C}(36)-\mathrm{C}(31)-\mathrm{C}(32 \mathrm{~A}) & 108.3(4) \\ \mathrm{C}(32 \mathrm{~B})-\mathrm{C}(31)-\mathrm{P}(1) & 121.7(8) \\ \mathrm{C}(36)-\mathrm{C}(31)-\mathrm{P}(1) & 118.2(2) \\ \mathrm{C}(32 \mathrm{~A})-\mathrm{C}(31)-\mathrm{P}(1) & 115.1(3) \\ \mathrm{C}(31)-\mathrm{C}(32 \mathrm{~A})-\mathrm{C}(33) & 107.5(4) \\ \mathrm{C}(31)-\mathrm{C}(32 \mathrm{~B})-\mathrm{C}(33) & 108.5(10) \\ \mathrm{C}(34)-\mathrm{C}(33)-\mathrm{C}(32 \mathrm{~A}) & 108.9(4) \\ \mathrm{C}(34)-\mathrm{C}(33)-\mathrm{C}(32 \mathrm{~B}) & 118.4(9) \\ \mathrm{C}(32 \mathrm{~A})-\mathrm{C}(33)-\mathrm{C}(32 \mathrm{~B}) & 48.3(12) \\ \mathrm{C}(35)-\mathrm{C}(34)-\mathrm{C}(33) & 112.3(5) \\ \mathrm{C}(34)-\mathrm{C}(35)-\mathrm{C}(36) & 114.4(4) \\ \mathrm{C}(31)-\mathrm{C}(36)-\mathrm{C}(35) & 111.6(3) \\ & \end{array}$


Symmetry transformations used to generate equivalent atoms:

\#1 -x,-y,-z 
Table 10. Anisotropic displacement parameters $\left(\AA^{2} \times 10^{3}\right)$ for $04014 \mathrm{IH}$ p-1. The Anisotropic displacement factor exponent takes the form: $-2 p^{2}\left[h^{2} a^{* 2} U^{11}+\ldots+2\right.$ $\mathrm{h} \mathrm{k} \mathrm{a}^{*} \mathrm{~b}^{*} \mathrm{U}^{12}$ ]

\begin{tabular}{lccccc}
\hline \multicolumn{1}{c}{$\mathrm{U}^{11}$} & $\mathrm{U} 22$ & $\mathrm{U} 33$ & $\mathrm{U} 23$ & $\mathrm{U} 13$ & $\mathrm{U} 12$ \\
\hline $\mathrm{Pd}(1) 45(1)$ & $97(1)$ & $31(1)$ & $-35(1)$ & $-17(1)$ & $37(1)$ \\
$\mathrm{P}(1) 29(1)$ & $62(1)$ & $43(1)$ & $-37(1)$ & $-9(1)$ & $8(1)$ \\
$\mathrm{C}(11) 38(2)$ & $56(2)$ & $39(2)$ & $-29(2)$ & $-7(2)$ & $1(2)$ \\
$\mathrm{C}(12) 50(2)$ & $74(3)$ & $38(2)$ & $-29(2)$ & $-3(2)$ & $4(2)$ \\
$\mathrm{C}(13) 60(3)$ & $81(3)$ & $40(2)$ & $-30(2)$ & $-5(2)$ & $-2(2)$ \\
$\mathrm{C}(14) 67(3)$ & $71(3)$ & $49(2)$ & $-17(2)$ & $-23(2)$ & $-12(2)$ \\
$\mathrm{C}(15) 66(3)$ & $73(3)$ & $68(3)$ & $-33(2)$ & $-32(2)$ & $17(2)$ \\
$\mathrm{C}(16) 60(2)$ & $65(2)$ & $56(2)$ & $-35(2)$ & $-20(2)$ & $11(2)$ \\
$\mathrm{C}(21) 37(2)$ & $53(2)$ & $41(2)$ & $-29(2)$ & $1(2)$ & $-2(2)$ \\
$\mathrm{C}(22) 47(2)$ & $63(2)$ & $54(2)$ & $-32(2)$ & $-12(2)$ & $4(2)$ \\
$\mathrm{C}(23) 52(2)$ & $57(2)$ & $59(2)$ & $-23(2)$ & $-8(2)$ & $1(2)$ \\
$\mathrm{C}(24) 58(2)$ & $53(2)$ & $56(2)$ & $-22(2)$ & $1(2)$ & $-7(2)$ \\
$\mathrm{C}(25) 71(3)$ & $59(2)$ & $59(2)$ & $-35(2)$ & $-5(2)$ & $-11(2)$ \\
$\mathrm{C}(26) 61(2)$ & $60(2)$ & $48(2)$ & $-30(2)$ & $-13(2)$ & $-6(2)$ \\
$\mathrm{C}(31) 36(2)$ & $66(2)$ & $84(3)$ & $-54(2)$ & $-21(2)$ & $13(2)$ \\
$\mathrm{C}(32 \mathrm{~A}) 50(3)$ & $73(3)$ & $113(5)$ & $-67(3)$ & $-44(3)$ & $26(2)$ \\
$\mathrm{C}(32 \mathrm{~B}) 77(11)$ & $151(15)$ & $145(17)$ & $-127(12)$ & $-34(9)$ & $10(10)$ \\
$\mathrm{C}(33) 65(3)$ & $123(4)$ & $278(8)$ & $-155(5)$ & $-77(4)$ & $32(3)$ \\
$\mathrm{C}(34) 56(3)$ & $95(4)$ & $232(7)$ & $-98(5)$ & $-59(4)$ & $14(3)$ \\
$\mathrm{C}(35) 36(2)$ & $103(4)$ & $153(5)$ & $-87(4)$ & $-17(3)$ & $5(2)$ \\
$\mathrm{C}(36) 36(2)$ & $68(3)$ & $90(3)$ & $-47(2)$ & $-11(2)$ & $3(2)$ \\
$\mathrm{Cl}(1 \mathrm{~A}) 37(1)$ & $82(1)$ & $34(1)$ & $-31(1)$ & $-11(1)$ & $3(1)$ \\
& & & & & \\
\hline
\end{tabular}


Table 11. Hydrogen coordinates ( $\times 10^{4}$ ) and isotropic displacement parameters $\left(\AA^{2} \times 10^{3}\right)$ for $04014 \mathrm{IH}$ p-1.

\begin{tabular}{lcccc}
\hline & $\mathrm{x}$ & $\mathrm{y}$ & $\mathrm{z}$ & $\mathrm{U}(\mathrm{eq})$ \\
\hline $\mathrm{H}(11)$ & -868 & -463 & 3676 & 52 \\
$\mathrm{H}(12 \mathrm{~A})$ & -3297 & -438 & 5060 & 67 \\
$\mathrm{H}(12 \mathrm{~B})$ & -3694 & -2098 & 5431 & 67 \\
$\mathrm{H}(13 \mathrm{~A})$ & -1636 & -1011 & 6285 & 75 \\
$\mathrm{H}(13 \mathrm{~B})$ & -3198 & -1945 & 7397 & 75 \\
$\mathrm{H}(14 \mathrm{~A})$ & -2483 & -3947 & 6991 & 78 \\
$\mathrm{H}(14 \mathrm{~B})$ & -1331 & -3375 & 7484 & 78 \\
$\mathrm{H}(15 \mathrm{~A})$ & -148 & -3948 & 5449 & 79 \\
$\mathrm{H}(15 \mathrm{~B})$ & 328 & -2297 & 5053 & 79 \\
$\mathrm{H}(16 \mathrm{~A})$ & -154 & -2386 & 3073 & 69 \\
$\mathrm{H}(16 \mathrm{~B})$ & -1728 & -3314 & 4153 & 69 \\
$\mathrm{H}(21)$ & -3483 & 1207 & 2865 & 54 \\
$\mathrm{H}(22 \mathrm{~A})$ & -1932 & 2445 & -262 & 65 \\
$\mathrm{H}(22 \mathrm{~B})$ & -3599 & 1695 & 553 & 65 \\
$\mathrm{H}(23 \mathrm{~A})$ & -3419 & 4253 & -417 & 72 \\
$\mathrm{H}(23 \mathrm{~B})$ & -4253 & 3535 & 1337 & 72 \\
$\mathrm{H}(24 \mathrm{~A})$ & -1257 & 4846 & -162 & 75 \\
$\mathrm{H}(24 \mathrm{~B})$ & -2534 & 5498 & 695 & 75 \\
$\mathrm{H}(25 \mathrm{~A})$ & -2839 & 3580 & 2954 & 78 \\
$\mathrm{H}(25 \mathrm{~B})$ & -1175 & 4338 & 2164 & 78 \\
$\mathrm{H}(26 \mathrm{~A})$ & -1328 & 1789 & 3129 & 67 \\
$\mathrm{H}(26 \mathrm{~B})$ & -485 & 2505 & 1377 & 67 \\
$\mathrm{H}(31 \mathrm{~A})$ & -3697 & -938 & 1242 & 66 \\
$\mathrm{H}(31 \mathrm{~B})$ & -3625 & -2198 & 3106 & 66 \\
$\mathrm{H}(32 \mathrm{~A})$ & -3855 & -3449 & 3757 & 79 \\
$\mathrm{H}(32 \mathrm{~B})$ & -2715 & -3108 & 2130 & 79 \\
$\mathrm{H}(32 \mathrm{C})$ & -2685 & -2506 & 1124 & 125 \\
$\mathrm{H}(32 \mathrm{D})$ & -3766 & -1549 & 448 & 125 \\
$\mathrm{H}(33 \mathrm{~A})$ & -4736 & -2965 & 1266 & 153 \\
$\mathrm{H}(33 \mathrm{~B})$ & -4907 & -4533 & 2548 & 153 \\
$\mathrm{H}(33 \mathrm{~A})$ & -5080 & -3855 & 1644 & 153 \\
& & & &
\end{tabular}




\begin{tabular}{lrrrr}
$\mathrm{H}(33 \mathrm{~B})$ & -4554 & -4274 & 2997 & 153 \\
$\mathrm{H}(34 \mathrm{~A})$ & -7130 & -3594 & 2984 & 139 \\
$\mathrm{H}(34 \mathrm{~B})$ & -6558 & -3894 & 4299 & 139 \\
$\mathrm{H}(35 \mathrm{~A})$ & -7313 & -1743 & 3746 & 110 \\
$\mathrm{H}(35 \mathrm{~B})$ & -6389 & -1225 & 2027 & 110 \\
$\mathrm{H}(36 \mathrm{~A})$ & -5165 & -88 & 2909 & 76 \\
$\mathrm{H}(36 \mathrm{~B})$ & -5231 & -1574 & 4246 & 76 \\
\hline
\end{tabular}


Table 12. Torsion angles [ $\left.{ }^{\circ}\right]$ for $04014 \mathrm{IH}$ p-1.

\begin{tabular}{lc}
\hline $\mathrm{P}(1) \# 1-\mathrm{Pd}(1)-\mathrm{P}(1)-\mathrm{C}(11)$ & $-100(100)$ \\
$\mathrm{Cl}(1 \mathrm{~A}) \# 1-\mathrm{Pd}(1)-\mathrm{P}(1)-\mathrm{C}(11)$ & $139.04(13)$ \\
$\mathrm{Cl}(1 \mathrm{~A})-\mathrm{Pd}(1)-\mathrm{P}(1)-\mathrm{C}(11)$ & $-40.96(13)$ \\
$\mathrm{P}(1) \# 1-\mathrm{Pd}(1)-\mathrm{P}(1)-\mathrm{C}(31)$ & $133(100)$ \\
$\mathrm{Cl}(1 \mathrm{~A}) \# 1-\mathrm{Pd}(1)-\mathrm{P}(1)-\mathrm{C}(31)$ & $12.67(15)$ \\
$\mathrm{Cl}(1 \mathrm{~A})-\mathrm{Pd}(1)-\mathrm{P}(1)-\mathrm{C}(31)$ & $-167.33(15)$ \\
$\mathrm{P}(1) \# 1-\mathrm{Pd}(1)-\mathrm{P}(1)-\mathrm{C}(21)$ & $16(100)$ \\
$\mathrm{Cl}(1 \mathrm{~A}) \# 1-\mathrm{Pd}(1)-\mathrm{P}(1)-\mathrm{C}(21)$ & $-104.47(13)$ \\
$\mathrm{Cl}(1 \mathrm{~A})-\mathrm{Pd}(1)-\mathrm{P}(1)-\mathrm{C}(21)$ & $75.53(13)$ \\
$\mathrm{C}(31)-\mathrm{P}(1)-\mathrm{C}(11)-\mathrm{C}(16)$ & $77.2(3)$ \\
$\mathrm{C}(21)-\mathrm{P}(1)-\mathrm{C}(11)-\mathrm{C}(16)$ & $-172.6(3)$ \\
$\mathrm{Pd}(1)-\mathrm{P}(1)-\mathrm{C}(11)-\mathrm{C}(16)$ & $-52.0(3)$ \\
$\mathrm{C}(31)-\mathrm{P}(1)-\mathrm{C}(11)-\mathrm{C}(12)$ & $-53.7(3)$ \\
$\mathrm{C}(21)-\mathrm{P}(1)-\mathrm{C}(11)-\mathrm{C}(12)$ & $56.5(3)$ \\
$\mathrm{Pd}(1)-\mathrm{P}(1)-\mathrm{C}(11)-\mathrm{C}(12)$ & $177.0(2)$ \\
$\mathrm{C}(16)-\mathrm{C}(11)-\mathrm{C}(12)-\mathrm{C}(13)$ & $57.8(4)$ \\
$\mathrm{P}(1)-\mathrm{C}(11)-\mathrm{C}(12)-\mathrm{C}(13)$ & $-169.7(3)$ \\
$\mathrm{C}(11)-\mathrm{C}(12)-\mathrm{C}(13)-\mathrm{C}(14)$ & $-57.1(4)$ \\
$\mathrm{C}(12)-\mathrm{C}(13)-\mathrm{C}(14)-\mathrm{C}(15)$ & $55.7(4)$ \\
$\mathrm{C}(13)-\mathrm{C}(14)-\mathrm{C}(15)-\mathrm{C}(16)$ & $-54.6(5)$ \\
$\mathrm{C}(12)-\mathrm{C}(11)-\mathrm{C}(16)-\mathrm{C}(15)$ & $-56.3(4)$ \\
$\mathrm{P}(1)-\mathrm{C}(11)-\mathrm{C}(16)-\mathrm{C}(15)$ & $170.2(3)$ \\
$\mathrm{C}(14)-\mathrm{C}(15)-\mathrm{C}(16)-\mathrm{C}(11)$ & $54.4(5)$ \\
$\mathrm{C}(11)-\mathrm{P}(1)-\mathrm{C}(21)-\mathrm{C}(26)$ & $53.7(3)$ \\
$\mathrm{C}(31)-\mathrm{P}(1)-\mathrm{C}(21)-\mathrm{C}(26)$ & $168.1(3)$ \\
$\mathrm{Pd}(1)-\mathrm{P}(1)-\mathrm{C}(21)-\mathrm{C}(26)$ & $-68.0(3)$ \\
$\mathrm{C}(11)-\mathrm{P}(1)-\mathrm{C}(21)-\mathrm{C}(22)$ & $178.0(2)$ \\
$\mathrm{C}(31)-\mathrm{P}(1)-\mathrm{C}(21)-\mathrm{C}(22)$ & $-67.6(3)$ \\
$\mathrm{Pd}(1)-\mathrm{P}(1)-\mathrm{C}(21)-\mathrm{C}(22)$ & $56.3(3)$ \\
$\mathrm{C}(26)-\mathrm{C}(21)-\mathrm{C}(22)-\mathrm{C}(23)$ & $-54.8(4)$ \\
$\mathrm{P}(1)-\mathrm{C}(21)-\mathrm{C}(22)-\mathrm{C}(23)$ & $179.1(2)$ \\
$\mathrm{C}(21)-\mathrm{C}(22)-\mathrm{C}(23)-\mathrm{C}(24)$ & $55.9(4)$ \\
$\mathrm{C}(22)-\mathrm{C}(23)-\mathrm{C}(24)-\mathrm{C}(25)$ & $-56.4(4)$ \\
$\mathrm{C}(23)-\mathrm{C}(24)-\mathrm{C}(25)-\mathrm{C}(26)$ & \\
&
\end{tabular}




$\begin{array}{lc}\mathrm{C}(24)-\mathrm{C}(25)-\mathrm{C}(26)-\mathrm{C}(21) & -56.6(4) \\ \mathrm{C}(22)-\mathrm{C}(21)-\mathrm{C}(26)-\mathrm{C}(25) & 54.7(4) \\ \mathrm{P}(1)-\mathrm{C}(21)-\mathrm{C}(26)-\mathrm{C}(25) & 179.0(2) \\ \mathrm{C}(11)-\mathrm{P}(1)-\mathrm{C}(31)-\mathrm{C}(32 \mathrm{~B}) & -113.4(17) \\ \mathrm{C}(21)-\mathrm{P}(1)-\mathrm{C}(31)-\mathrm{C}(32 \mathrm{~B}) & 136.0(17) \\ \mathrm{Pd}(1)-\mathrm{P}(1)-\mathrm{C}(31)-\mathrm{C}(32 \mathrm{~B}) & 14.7(17) \\ \mathrm{C}(11)-\mathrm{P}(1)-\mathrm{C}(31)-\mathrm{C}(36) & 74.4(4) \\ \mathrm{C}(21)-\mathrm{P}(1)-\mathrm{C}(31)-\mathrm{C}(36) & -36.2(4) \\ \mathrm{Pd}(1)-\mathrm{P}(1)-\mathrm{C}(31)-\mathrm{C}(36) & -157.5(3) \\ \mathrm{C}(11)-\mathrm{P}(1)-\mathrm{C}(31)-\mathrm{C}(32 \mathrm{~A}) & -55.6(4) \\ \mathrm{C}(21)-\mathrm{P}(1)-\mathrm{C}(31)-\mathrm{C}(32 \mathrm{~A}) & -166.2(4) \\ \mathrm{Pd}(1)-\mathrm{P}(1)-\mathrm{C}(31)-\mathrm{C}(32 \mathrm{~A}) & 72.5(4) \\ \mathrm{C}(32 \mathrm{~B})-\mathrm{C}(31)-\mathrm{C}(32 \mathrm{~A})-\mathrm{C}(33) & -51.1(11) \\ \mathrm{C}(36)-\mathrm{C}(31)-\mathrm{C}(32 \mathrm{~A})-\mathrm{C}(33) & 62.7(6) \\ \mathrm{P}(1)-\mathrm{C}(31)-\mathrm{C}(32 \mathrm{~A})-\mathrm{C}(33) & -162.6(4) \\ \mathrm{C}(36)-\mathrm{C}(31)-\mathrm{C}(32 \mathrm{~B})-\mathrm{C}(33) & -39(3) \\ \mathrm{C}(32 \mathrm{~A})-\mathrm{C}(31)-\mathrm{C}(32 \mathrm{~B})-\mathrm{C}(33) & 50.9(14) \\ \mathrm{P}(1)-\mathrm{C}(31)-\mathrm{C}(32 \mathrm{~B})-\mathrm{C}(33) & 148.8(12) \\ \mathrm{C}(31)-\mathrm{C}(32 \mathrm{~A})-\mathrm{C}(33)-\mathrm{C}(34) & -62.2(7) \\ \mathrm{C}(31)-\mathrm{C}(32 \mathrm{~A})-\mathrm{C}(33)-\mathrm{C}(32 \mathrm{~B}) & 49.3(11) \\ \mathrm{C}(31)-\mathrm{C}(32 \mathrm{~B})-\mathrm{C}(33)-\mathrm{C}(34) & 39(3) \\ \mathrm{C}(31)-\mathrm{C}(32 \mathrm{~B})-\mathrm{C}(33)-\mathrm{C}(32 \mathrm{~A}) & -51.2(14) \\ \mathrm{C}(32 \mathrm{~A})-\mathrm{C}(33)-\mathrm{C}(34)-\mathrm{C}(35) & 56.2(8) \\ \mathrm{C}(32 \mathrm{~B})-\mathrm{C}(33)-\mathrm{C}(34)-\mathrm{C}(35) & 4.1(16) \\ \mathrm{C}(33)-\mathrm{C}(34)-\mathrm{C}(35)-\mathrm{C}(36) & -51.3(8) \\ \mathrm{C}(32 \mathrm{~B})-\mathrm{C}(31)-\mathrm{C}(36)-\mathrm{C}(35) & -2.6(16) \\ \mathrm{C}(32 \mathrm{~A})-\mathrm{C}(31)-\mathrm{C}(36)-\mathrm{C}(35) & -57.1(5) \\ \mathrm{P}(1)-\mathrm{C}(31)-\mathrm{C}(36)-\mathrm{C}(35) & 169.7(4) \\ \mathrm{C}(34)-\mathrm{C}(35)-\mathrm{C}(36)-\mathrm{C}(31) & 51.8(7) \\ & \\ & \\ & \\ & \end{array}$

Symmetry transformations used to generate equivalent atoms: \#1 -x,-y,-z 
<smiles>CCCCC(C)(C)C</smiles>

2

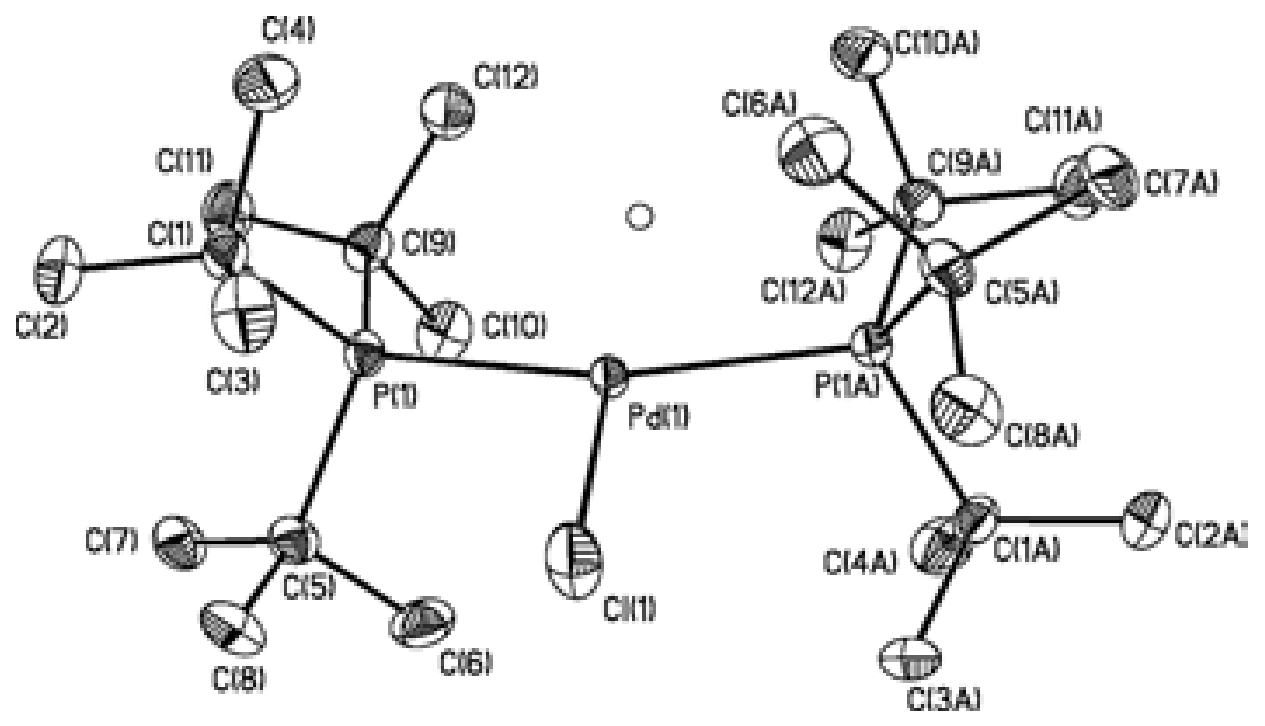

A light-gray solution of 2 in $\mathrm{Et}_{2} \mathrm{O}$ was prepared. Crystals suitable for X-ray structural analysis were obtained by diffusing pentanes into the $\mathrm{Et}_{2} \mathrm{O}$ solution.

A colorless plate of dimensions $0.27 \times 0.26 \times 0.07 \mathrm{~mm}^{3}$ was mounted under STP and transferred to a Bruker AXS/CCD three-circle diffractometer ( $\chi$ fixed at 54.78 ${ }^{\circ}$ equipped with a cold stream of $\mathrm{N}_{2}$ gas. An initial unit cell was determined by harvesting reflections I > 20 oI) from $45 \times 10$-s frames of $0.30^{\circ} \omega$ scan data with monochromated Mo $\mathrm{K}_{\alpha}$ radiation $(\lambda=0.71073 \AA$ ) $)$. The cell thus determined was monoclinic.

A hemisphere of data was then collected using $\omega$ scans of $0.30^{\circ}$ and 30 -s frames. The raw data frames were integrated using the Bruker program SAINT+ for NT version 6.01. The data that were collected (5946 total reflections, 2015 unique, $\mathrm{R}_{\text {int }}=$ 
0.0366) had the following Miller index ranges: -12 to 14 in $h,-9$ to 7 in $k$, and -15 to 15 in 1. No absorption correction was performed.

All aspects of the solution and refinement were handled by SHELXTL NT version 5.10. The structure was solved by direct methods in the monoclinic space group $\mathrm{P} 2 / \mathrm{n}$, a $=12.7692(12) \AA ; b=8.7306(9) \AA ; c=13.8817(14) \AA ; \alpha=90^{\circ} ; \beta=114.718(2)^{\circ} ; \gamma=90^{\circ}$, and refined using standard difference Fourier techniques. Final, full-matrix least-squares refinement (2015 data for 139 parameters) on $\mathrm{F}^{2}$ yielded residuals of $\mathrm{R}_{1}$ and $w \mathrm{R}_{2}$ of 0.0475 and 0.1198 for data $\mathrm{I}>2 \sigma(\mathrm{I})$, and 0.0511 and 0.1237 , respectively, for all data. During the final refinement, all non-hydrogen atoms were treated anisotropically. Hydrogen atoms were included in calculated positions and refined isotropically on a riding model. Residual electron density amounted to a maximum of $3.011 \mathrm{e} / \AA^{3}$ and a minimum of $-0.357 \mathrm{e} / \AA^{3}$.

The metal-hydride has not been located and is represented graphically in Figure 2 merely for illustrative purposes. 
Tables 1-6 provide the full crystallographic data for the X-ray structure.

Table 1. Crystal data and structure refinement for 04006mih.

Identification code

Empirical formula

Formula weight

Temperature

Wavelength

Crystal system

Space group

Unit cell dimensions

Volume

Z

Density (calculated)

Absorption coefficient

$\mathrm{F}(000)$

Crystal size

Theta range for data collection

Index ranges

Reflections collected

Independent reflections

Completeness to theta $=23.25^{\circ}$

Absorption correction

Refinement method

Data / restraints / parameters

Goodness-of-fit on F 2

Final $\mathrm{R}$ indices [I $>2$ sigma(I)]

$\mathrm{R}$ indices (all data)

Largest diff. peak and hole 04006mih

C24 H55 Cl P2 Pd

547.47

193(2) K

$0.71073 \AA$

Monoclinic

$\mathrm{P} 2 / \mathrm{n}$

$$
\begin{array}{ll}
\mathrm{a}=12.7692(12) \AA & \alpha=90^{\circ} . \\
\mathrm{b}=8.7306(9) \AA & \beta=114.718(2)^{\circ} . \\
\mathrm{c}=13.8817(14) \AA & \gamma=90^{\circ} .
\end{array}
$$

\section{$1405.8(2) \AA^{3}$}

2

$1.293 \mathrm{Mg} / \mathrm{m}^{3}$

$0.878 \mathrm{~mm}^{-1}$

584

$0.27 \times 0.26 \times 0.07 \mathrm{~mm}^{3}$

1.82 to $23.25^{\circ}$.

$-12<=\mathrm{h}<=14,-9<=\mathrm{k}<=7,-15<=\mathrm{l}<=15$

5946

$2015[\mathrm{R}$ (int) $=0.0366]$

$99.7 \%$

None

Full-matrix least-squares on $\mathrm{F}^{2}$

2015 / 0 / 139

1.059

$\mathrm{R} 1=0.0475, \mathrm{wR} 2=0.1198$

$\mathrm{R} 1=0.0511, \mathrm{wR} 2=0.1237$

3.011 and -0.357 e. $\AA^{-3}$ 
Table 2. Atomic coordinates $\left(\times 10^{4}\right)$ and equivalent isotropic displacement parameters $\left(\AA^{2} \times 10^{3}\right)$ for $04006 \mathrm{mih} . \mathrm{U}(\mathrm{eq})$ is defined as one third of the trace of the orthogonalized $\mathrm{U}^{\mathrm{ij}}$ tensor.

\begin{tabular}{lllll}
\hline & \multicolumn{1}{c}{$\mathrm{x}$} & \multicolumn{1}{c}{$\mathrm{y}$} & \multicolumn{1}{c}{$\mathrm{z}$} & $\mathrm{U}(\mathrm{eq})$ \\
\hline $\mathrm{Pd}(1)$ & 2500 & $7501(1)$ & 2500 & $19(1)$ \\
$\mathrm{P}(1)$ & $4191(1)$ & $7955(1)$ & $2249(1)$ & $24(1)$ \\
$\mathrm{Cl}(1)$ & 2500 & $4702(2)$ & 2500 & $49(1)$ \\
$\mathrm{C}(1)$ & $4088(4)$ & $7299(5)$ & $902(4)$ & $29(1)$ \\
$\mathrm{C}(2)$ & $5223(5)$ & $7185(6)$ & $795(4)$ & $41(1)$ \\
$\mathrm{C}(3)$ & $3495(4)$ & $5714(6)$ & $646(4)$ & $43(1)$ \\
$\mathrm{C}(4)$ & $3264(4)$ & $8368(7)$ & $33(3)$ & $47(1)$ \\
$\mathrm{C}(5)$ & $5446(3)$ & $6931(6)$ & $3344(3)$ & $32(1)$ \\
$\mathrm{C}(6)$ & $5235(5)$ & $7044(7)$ & $4365(4)$ & $46(1)$ \\
$\mathrm{C}(7)$ & $6662(4)$ & $7552(5)$ & $3602(4)$ & $39(1)$ \\
$\mathrm{C}(8)$ & $5444(4)$ & $5215(5)$ & $3089(4)$ & $42(1)$ \\
$\mathrm{C}(9)$ & $4538(4)$ & $10133(5)$ & $2352(3)$ & $32(1)$ \\
$\mathrm{C}(10)$ & $4841(4)$ & $10635(5)$ & $3502(4)$ & $43(1)$ \\
$\mathrm{C}(11)$ & $5536(4)$ & $10617(6)$ & $2069(4)$ & $45(1)$ \\
$\mathrm{C}(12)$ & $3475(4)$ & $11085(5)$ & $1642(4)$ & $43(1)$ \\
& & & & \\
\hline
\end{tabular}


Table 3. Bond lengths $[\AA]$ and angles $\left[{ }^{\circ}\right]$ for $04006 \mathrm{mih}$.

\begin{tabular}{lc}
\hline $\mathrm{Pd}(1)-\mathrm{P}(1) \# 1$ & $2.3608(11)$ \\
$\mathrm{Pd}(1)-\mathrm{P}(1)$ & $2.3608(11)$ \\
$\mathrm{Pd}(1)-\mathrm{Cl}(1)$ & $2.4436(18)$ \\
$\mathrm{P}(1)-\mathrm{C}(1)$ & $1.906(5)$ \\
$\mathrm{P}(1)-\mathrm{C}(5)$ & $1.907(4)$ \\
$\mathrm{P}(1)-\mathrm{C}(9)$ & $1.944(4)$ \\
$\mathrm{C}(1)-\mathrm{C}(2)$ & $1.520(8)$ \\
$\mathrm{C}(1)-\mathrm{C}(4)$ & $1.540(6)$ \\
$\mathrm{C}(1)-\mathrm{C}(3)$ & $1.546(6)$ \\
$\mathrm{C}(5)-\mathrm{C}(8)$ & $1.539(7)$ \\
$\mathrm{C}(5)-\mathrm{C}(7)$ & $1.540(7)$ \\
$\mathrm{C}(5)-\mathrm{C}(6)$ & $1.552(7)$ \\
$\mathrm{C}(9)-\mathrm{C}(11)$ & $1.542(6)$ \\
$\mathrm{C}(9)-\mathrm{C}(10)$ & $1.541(6)$ \\
$\mathrm{C}(9)-\mathrm{C}(12)$ & $1.544(6)$ \\
$\mathrm{P}(1) \# 1-\mathrm{Pd}(1)-\mathrm{P}(1)$ & $160.67(6)$ \\
$\mathrm{P}(1) \# 1-\mathrm{Pd}(1)-\mathrm{Cl}(1)$ & $99.67(3)$ \\
$\mathrm{P}(1)-\mathrm{Pd}(1)-\mathrm{Cl}(1)$ & $99.67(3)$ \\
$\mathrm{C}(1)-\mathrm{P}(1)-\mathrm{C}(5)$ & $109.4(2)$ \\
$\mathrm{C}(1)-\mathrm{P}(1)-\mathrm{C}(9)$ & $106.62(18)$ \\
$\mathrm{C}(5)-\mathrm{P}(1)-\mathrm{C}(9)$ & $108.1(2)$ \\
$\mathrm{C}(1)-\mathrm{P}(1)-\mathrm{Pd}(1)$ & $113.71(15)$ \\
$\mathrm{C}(5)-\mathrm{P}(1)-\mathrm{Pd}(1)$ & $108.59(14)$ \\
$\mathrm{C}(9)-\mathrm{P}(1)-\mathrm{Pd}(1)$ & $110.33(14)$ \\
$\mathrm{C}(2)-\mathrm{C}(1)-\mathrm{C}(4)$ & $109.3(4)$ \\
$\mathrm{C}(2)-\mathrm{C}(1)-\mathrm{C}(3)$ & $108.3(4)$ \\
$\mathrm{C}(4)-\mathrm{C}(1)-\mathrm{C}(3)$ & $104.7(4)$ \\
$\mathrm{C}(2)-\mathrm{C}(1)-\mathrm{P}(1)$ & $116.0(3)$ \\
$\mathrm{C}(4)-\mathrm{C}(1)-\mathrm{P}(1)$ & $109.3(3)$ \\
$\mathrm{C}(3)-\mathrm{C}(1)-\mathrm{P}(1)$ & $108.7(3)$ \\
$\mathrm{C}(8)-\mathrm{C}(5)-\mathrm{C}(7)$ & $107.5(4)$ \\
$\mathrm{C}(8)-\mathrm{C}(5)-\mathrm{C}(6)$ & $106.7(4)$ \\
$\mathrm{C}(7)-\mathrm{C}(5)-\mathrm{C}(6)$ & $108.0(4)$ \\
$\mathrm{C}(8)-\mathrm{C}(5)-\mathrm{P}(1)$ & $110.7(3)$ \\
&
\end{tabular}




$\begin{array}{ll}\mathrm{C}(7)-\mathrm{C}(5)-\mathrm{P}(1) & 116.7(3) \\ \mathrm{C}(6)-\mathrm{C}(5)-\mathrm{P}(1) & 106.8(3) \\ \mathrm{C}(11)-\mathrm{C}(9)-\mathrm{C}(10) & 108.0(4) \\ \mathrm{C}(11)-\mathrm{C}(9)-\mathrm{C}(12) & 106.8(4) \\ \mathrm{C}(10)-\mathrm{C}(9)-\mathrm{C}(12) & 106.3(4) \\ \mathrm{C}(11)-\mathrm{C}(9)-\mathrm{P}(1) & 115.9(3) \\ \mathrm{C}(10)-\mathrm{C}(9)-\mathrm{P}(1) & 107.8(3) \\ \mathrm{C}(12)-\mathrm{C}(9)-\mathrm{P}(1) & 111.6(3)\end{array}$

Symmetry transformations used to generate equivalent atoms:

$\# 1-x+1 / 2, y,-z+1 / 2$ 
Table 4. Anisotropic displacement parameters $\left(\AA^{2} \times 10^{3}\right)$ for $04006 \mathrm{mih}$. The anisotropic displacement factor exponent takes the form: $-2 p^{2}\left[h^{2} a^{* 2} U^{11}+\ldots+2 h k\right.$ $\left.a^{*} b^{*} U^{12}\right]$.

\begin{tabular}{|c|c|c|c|c|c|}
\hline $\mathrm{U}^{11}$ & $\mathrm{U}^{22}$ & $\mathrm{U}^{33}$ & $\mathrm{U}^{23}$ & $\mathrm{U}^{13}$ & $\mathrm{U}^{12}$ \\
\hline $\operatorname{Pd}(1) 18(1)$ & $16(1)$ & $25(1)$ & 0 & $11(1)$ & 0 \\
\hline $\mathrm{P}(1) \quad 20(1)$ & $29(1)$ & $24(1)$ & $-2(1)$ & $10(1)$ & $-1(1)$ \\
\hline $\mathrm{Cl}(1) 52(1)$ & $21(1)$ & 92(1) & 0 & $48(1)$ & 0 \\
\hline$C(1) 28(3)$ & $38(3)$ & $24(2)$ & $-2(2)$ & $13(2)$ & $3(2)$ \\
\hline$C(2) 40(3)$ & $52(3)$ & $41(3)$ & $-9(2)$ & $26(2)$ & $-2(2)$ \\
\hline$C(3) 41(3)$ & $43(3)$ & $45(3)$ & $-24(2)$ & $17(2)$ & $-10(2)$ \\
\hline$C(4) 48(3)$ & $59(4)$ & $30(2)$ & $1(2)$ & $12(2)$ & $6(3)$ \\
\hline$C(5) \quad 22(2)$ & $36(3)$ & $32(2)$ & $4(2)$ & $7(2)$ & $-1(2)$ \\
\hline C(6) 48(3) & $57(3)$ & $29(2)$ & $11(2)$ & $11(2)$ & 1(3) \\
\hline$C(7) 21(3)$ & $47(4)$ & $39(3)$ & $3(2)$ & $5(2)$ & $2(2)$ \\
\hline C(8) 34(3) & $33(3)$ & $51(3)$ & $6(2)$ & $10(2)$ & $10(2)$ \\
\hline$C(9) 39(2)$ & $24(2)$ & $37(2)$ & $-1(2)$ & $20(2)$ & $-6(2)$ \\
\hline$C(10) 50(3)$ & $32(3)$ & $49(3)$ & $-12(2)$ & $24(2)$ & $-13(2)$ \\
\hline$C(11) 45(3)$ & $38(3)$ & $62(3)$ & $-3(2)$ & $31(2)$ & $-6(2)$ \\
\hline$C(12) 51(3)$ & $29(3)$ & $58(3)$ & $7(2)$ & $31(2)$ & $5(2)$ \\
\hline
\end{tabular}


Table 5. Hydrogen coordinates $\left(x 10^{4}\right)$ and isotropic displacement parameters $\left(\AA^{2} x\right.$ $10^{3}$ ) for 04006mih.

\begin{tabular}{|c|c|c|c|c|}
\hline & $x$ & $\mathrm{y}$ & z & $\mathrm{U}(\mathrm{eq})$ \\
\hline $\mathrm{H}(2 \mathrm{~A})$ & 5072 & 6886 & 69 & 62 \\
\hline $\mathrm{H}(2 \mathrm{~B})$ & 5715 & 6414 & 1292 & 62 \\
\hline $\mathrm{H}(2 \mathrm{C})$ & 5613 & 8180 & 956 & 62 \\
\hline $\mathrm{H}(3 \mathrm{~A})$ & 3368 & 5410 & -74 & 65 \\
\hline $\mathrm{H}(3 \mathrm{~B})$ & 2754 & 5770 & 697 & 65 \\
\hline $\mathrm{H}(3 \mathrm{C})$ & 3989 & 4956 & 1154 & 65 \\
\hline $\mathrm{H}(4 \mathrm{~A})$ & 3625 & 9375 & 94 & 70 \\
\hline $\mathrm{H}(4 \mathrm{~B})$ & 2544 & 8478 & 118 & 70 \\
\hline $\mathrm{H}(4 \mathrm{C})$ & 3099 & 7931 & -666 & 70 \\
\hline $\mathrm{H}(6 \mathrm{~A})$ & 5859 & 6520 & 4948 & 70 \\
\hline $\mathrm{H}(6 \mathrm{~B})$ & 4497 & 6560 & 4241 & 70 \\
\hline $\mathrm{H}(6 \mathrm{C})$ & 5216 & 8124 & 4550 & 70 \\
\hline $\mathrm{H}(7 \mathrm{~A})$ & 6726 & 8603 & 3868 & 58 \\
\hline $\mathrm{H}(7 \mathrm{~B})$ & 6800 & 7544 & 2958 & 58 \\
\hline $\mathrm{H}(7 \mathrm{C})$ & 7236 & 6905 & 4143 & 58 \\
\hline $\mathrm{H}(8 \mathrm{~A})$ & 5676 & 5082 & 2505 & 63 \\
\hline $\mathrm{H}(8 \mathrm{~B})$ & 4668 & 4797 & 2883 & 63 \\
\hline $\mathrm{H}(8 \mathrm{C})$ & 5988 & 4673 & 3718 & 63 \\
\hline $\mathrm{H}(10 \mathrm{~A})$ & 5563 & 10146 & 3979 & 64 \\
\hline $\mathrm{H}(10 \mathrm{~B})$ & 4221 & 10328 & 3702 & 64 \\
\hline $\mathrm{H}(10 \mathrm{C})$ & 4932 & 11750 & 3557 & 64 \\
\hline $\mathrm{H}(11 \mathrm{~A})$ & 5319 & 10431 & 1313 & 68 \\
\hline $\mathrm{H}(11 \mathrm{~B})$ & 6226 & 10019 & 2491 & 68 \\
\hline $\mathrm{H}(11 \mathrm{C})$ & 5698 & 11709 & 2222 & 68 \\
\hline $\mathrm{H}(12 \mathrm{~A})$ & 3627 & 12173 & 1819 & 65 \\
\hline $\mathrm{H}(12 \mathrm{~B})$ & 2804 & 10760 & 1761 & 65 \\
\hline $\mathrm{H}(12 \mathrm{C})$ & 3320 & 10926 & 896 & 65 \\
\hline $\mathrm{H}(99)$ & 2500 & 10310(50) & 2500 & $0(11)$ \\
\hline
\end{tabular}


Table 6. Torsion angles $\left[{ }^{\circ}\right]$ for $04006 \mathrm{mih}$.

\begin{tabular}{lc}
\hline $\mathrm{P}(1) \# 1-\mathrm{Pd}(1)-\mathrm{P}(1)-\mathrm{C}(1)$ & $-114.05(15)$ \\
$\mathrm{Cl}(1)-\mathrm{Pd}(1)-\mathrm{P}(1)-\mathrm{C}(1)$ & $65.95(15)$ \\
$\mathrm{P}(1) \# 1-\mathrm{Pd}(1)-\mathrm{P}(1)-\mathrm{C}(5)$ & $123.95(16)$ \\
$\mathrm{Cl}(1)-\mathrm{Pd}(1)-\mathrm{P}(1)-\mathrm{C}(5)$ & $-56.05(16)$ \\
$\mathrm{P}(1) \# 1-\mathrm{Pd}(1)-\mathrm{P}(1)-\mathrm{C}(9)$ & $5.68(14)$ \\
$\mathrm{Cl}(1)-\mathrm{Pd}(1)-\mathrm{P}(1)-\mathrm{C}(9)$ & $-174.32(14)$ \\
$\mathrm{C}(5)-\mathrm{P}(1)-\mathrm{C}(1)-\mathrm{C}(2)$ & $-43.5(4)$ \\
$\mathrm{C}(9)-\mathrm{P}(1)-\mathrm{C}(1)-\mathrm{C}(2)$ & $73.1(4)$ \\
$\mathrm{Pd}(1)-\mathrm{P}(1)-\mathrm{C}(1)-\mathrm{C}(2)$ & $-165.1(3)$ \\
$\mathrm{C}(5)-\mathrm{P}(1)-\mathrm{C}(1)-\mathrm{C}(4)$ & $-167.6(3)$ \\
$\mathrm{C}(9)-\mathrm{P}(1)-\mathrm{C}(1)-\mathrm{C}(4)$ & $-50.9(4)$ \\
$\mathrm{Pd}(1)-\mathrm{P}(1)-\mathrm{C}(1)-\mathrm{C}(4)$ & $70.9(3)$ \\
$\mathrm{C}(5)-\mathrm{P}(1)-\mathrm{C}(1)-\mathrm{C}(3)$ & $78.7(3)$ \\
$\mathrm{C}(9)-\mathrm{P}(1)-\mathrm{C}(1)-\mathrm{C}(3)$ & $-164.7(3)$ \\
$\mathrm{Pd}(1)-\mathrm{P}(1)-\mathrm{C}(1)-\mathrm{C}(3)$ & $-42.9(3)$ \\
$\mathrm{C}(1)-\mathrm{P}(1)-\mathrm{C}(5)-\mathrm{C}(8)$ & $-44.7(4)$ \\
$\mathrm{C}(9)-\mathrm{P}(1)-\mathrm{C}(5)-\mathrm{C}(8)$ & $-160.4(3)$ \\
$\mathrm{Pd}(1)-\mathrm{P}(1)-\mathrm{C}(5)-\mathrm{C}(8)$ & $79.9(3)$ \\
$\mathrm{C}(1)-\mathrm{P}(1)-\mathrm{C}(5)-\mathrm{C}(7)$ & $78.6(4)$ \\
$\mathrm{C}(9)-\mathrm{P}(1)-\mathrm{C}(5)-\mathrm{C}(7)$ & $-37.1(4)$ \\
$\mathrm{Pd}(1)-\mathrm{P}(1)-\mathrm{C}(5)-\mathrm{C}(7)$ & $-156.8(3)$ \\
$\mathrm{C}(1)-\mathrm{P}(1)-\mathrm{C}(5)-\mathrm{C}(6)$ & $-160.5(3)$ \\
$\mathrm{C}(9)-\mathrm{P}(1)-\mathrm{C}(5)-\mathrm{C}(6)$ & $83.8(3)$ \\
$\mathrm{Pd}(1)-\mathrm{P}(1)-\mathrm{C}(5)-\mathrm{C}(6)$ & $-35.9(4)$ \\
$\mathrm{C}(1)-\mathrm{P}(1)-\mathrm{C}(9)-\mathrm{C}(11)$ & $-48.9(4)$ \\
$\mathrm{C}(5)-\mathrm{P}(1)-\mathrm{C}(9)-\mathrm{C}(11)$ & $68.6(4)$ \\
$\mathrm{Pd}(1)-\mathrm{P}(1)-\mathrm{C}(9)-\mathrm{C}(11)$ & $-172.8(3)$ \\
$\mathrm{C}(1)-\mathrm{P}(1)-\mathrm{C}(9)-\mathrm{C}(10)$ & $-170.0(3)$ \\
$\mathrm{C}(5)-\mathrm{P}(1)-\mathrm{C}(9)-\mathrm{C}(10)$ & $-52.5(3)$ \\
$\mathrm{Pd}(1)-\mathrm{P}(1)-\mathrm{C}(9)-\mathrm{C}(10)$ & $66.1(3)$ \\
$\mathrm{C}(1)-\mathrm{P}(1)-\mathrm{C}(9)-\mathrm{C}(12)$ & $73.6(3)$ \\
$\mathrm{C}(5)-\mathrm{P}(1)-\mathrm{C}(9)-\mathrm{C}(12)$ & $-168.9(3)$ \\
$\mathrm{Pd}(1)-\mathrm{P}(1)-\mathrm{C}(9)-\mathrm{C}(12)$ & $-50.3(3)$ \\
& \\
\hline
\end{tabular}


Symmetry transformations used to generate equivalent atoms:

\#1-x+1/2,y,-z+1/2 